University of North Florida

UNIVERSITY of

NORTH FLORIDA.

UNF Digital Commons

\title{
Total Brachial Artery Reactivity and Incident Heart Failure and Heart Failure Sub-Types: Multi-Ethnic Study of Atherosclerosis
}

Daniela Charry Segura

University of North Florida, n01447470@unf.edu

Follow this and additional works at: https://digitalcommons.unf.edu/etd

Part of the Cardiovascular Diseases Commons

\section{Suggested Citation}

Charry Segura, Daniela, "Total Brachial Artery Reactivity and Incident Heart Failure and Heart Failure SubTypes: Multi-Ethnic Study of Atherosclerosis" (2021). UNF Graduate Theses and Dissertations. 1008. https://digitalcommons.unf.edu/etd/1008

This Master's Thesis is brought to you for free and open access by the Student Scholarship at UNF Digital Commons. It has been accepted for inclusion in UNF Graduate Theses and Dissertations by an authorized administrator of UNF Digital Commons. For more information, please contact Digital Projects.

(c) 2021 All Rights Reserved

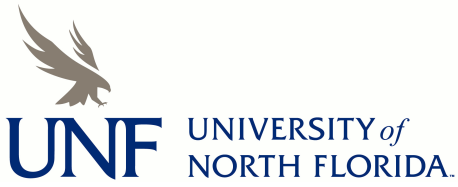


TOTAL BRACHIAL ARTERY REACTIVITY AND INCIDENT HEART FAILURE AND HEART FAILURE SUB-TYPES: MULTI-ETHNIC STUDY OF ATHEROSCLEROSIS

By

Daniela Charry Segura

A thesis submitted to the Department of Clinical and Applied Movement Sciences in partial fulfillment of the requirements for the degree of Master of Science in Health, Exercise Science and Chronic Disease

UNIVERSITY OF NORTH FLORIDA

BROOKS COLLEGE OF HEALTH

April, 2021 
The thesis of Daniela Charry Segura is approved:

(Date)

Dr. James R. Churilla, PhD, MPH, MS, RCEP, FACSM Committee Chairperson

Dr. Michael R. Richardson, PhD, MSH, ACSM EP-C

Committee Member

Dr. Jasper Xu, PhD, MHA, CHFP

Committee Member

Dr. Hirofumi Tanaka, PhD

Committee Member

Dr. Kevin S. Heffernan, PhD, FACSM

Committee Member 


\section{Dedication and Acknowledgements}

Every challenging work requires self-efforts as well as guidance and support. This thesis is dedicated to my family, friends, and all the people who have helped me during the past twoyears. I owe my greatest gratitude to my mom for her encouragement and words of wisdom that kept me focused and led me to successfully achieve my goals and to the Baldwin family, who have supported me in so many ways. There are no words to express my appreciation and love for you.

I wish to thank my committee members, Dr. James Churilla, Dr. Jasper Xu, Dr. Hirofumi Tanaka, Dr. Kevin Heffernan, and Dr. Ryan Richardson who generously provided me their time and expertise. Thank you for your constant assistance and feedback throughout the process which allowed me to successfully complete this thesis. A special thanks to Dr. James Churilla for being my mentor during graduate school. I appreciate your guidance, advice, and all the academic opportunities you provided me during my time at the University of North Florida. Thank you for your patience and trust.

Finally, I would like to thank my fellow graduate research assistants Chakene Rogers, Michelle Stone, and Bethany Rand for your friendship, assistance, and support. Thank you to my friends, especially Angela Mateus and Leidy Molano, whose words and sense of humor kept me sane during hard times. 


\section{Table of Contents}

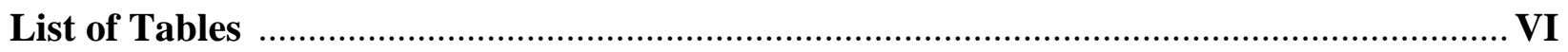

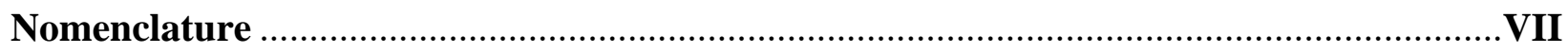

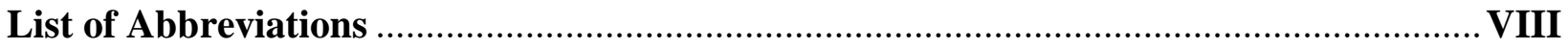

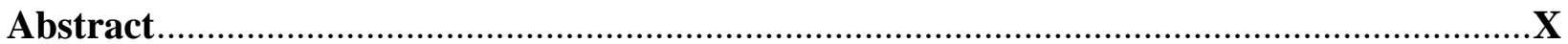

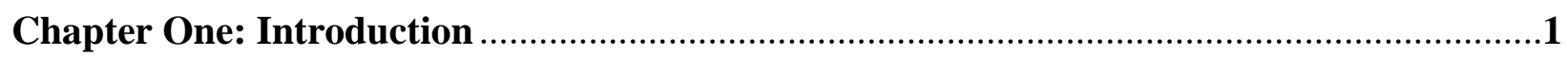

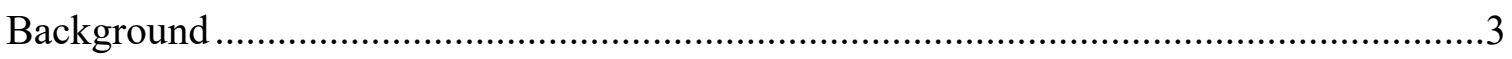

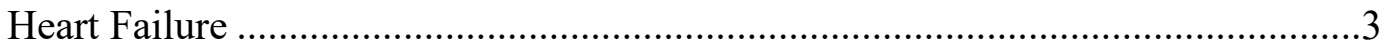

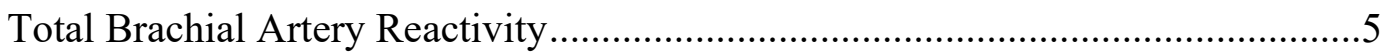

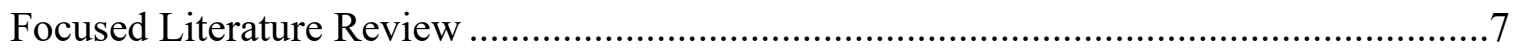

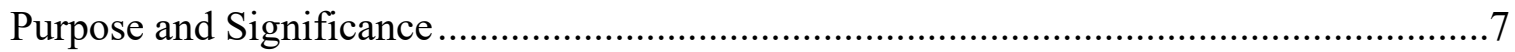

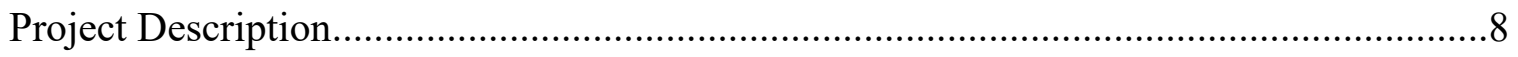

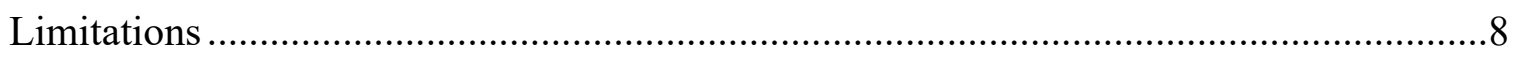

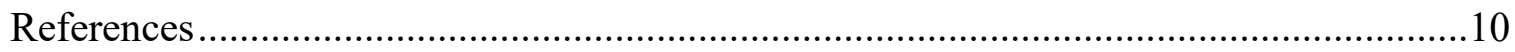

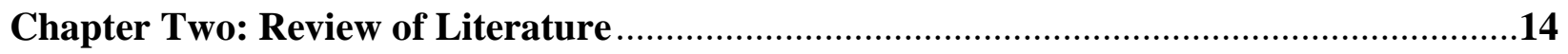

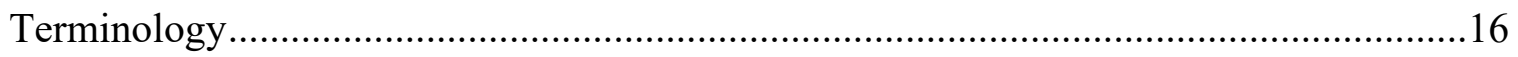

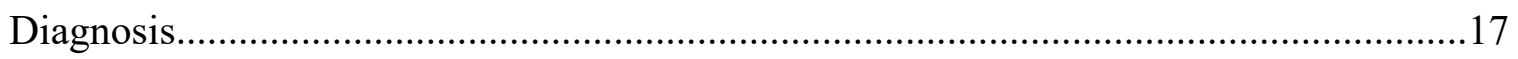

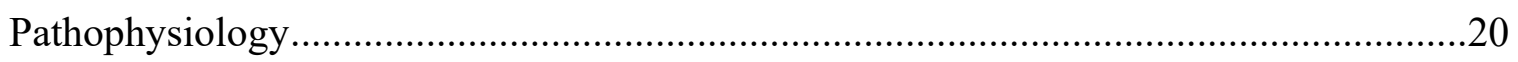

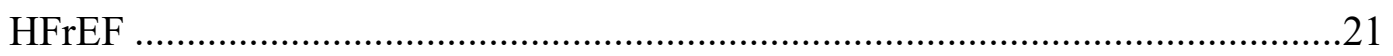

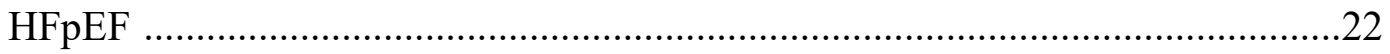

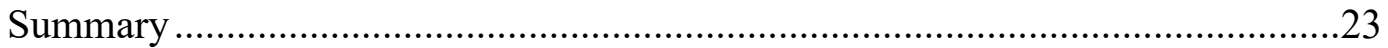

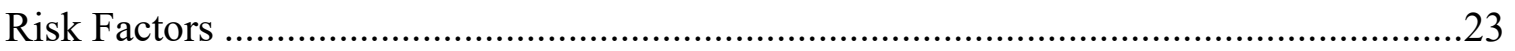

Heart Failure and Endothelial Dysfunction ...............................................................28

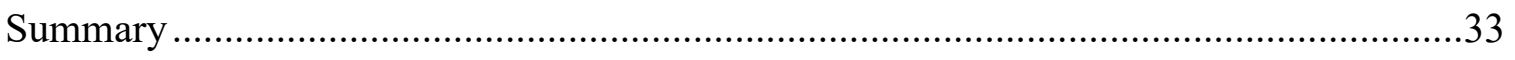

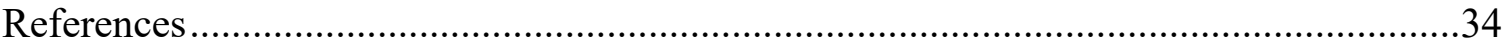

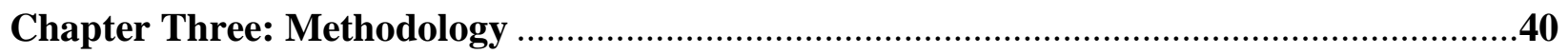

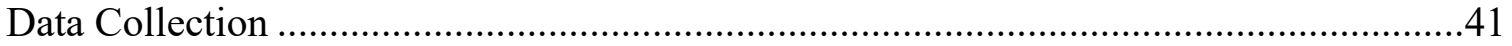

Primary Dependent Variable: Heart Failure ………………………………………....43

Primary Independent Variable: TBAR …………………………………………......4

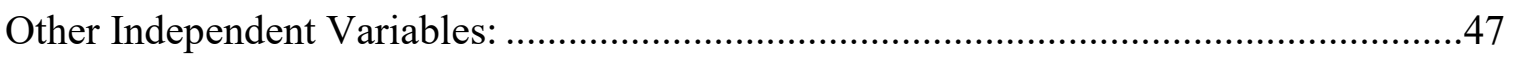

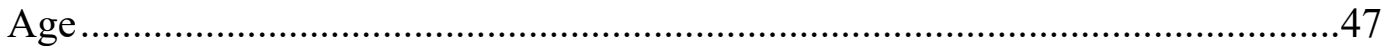




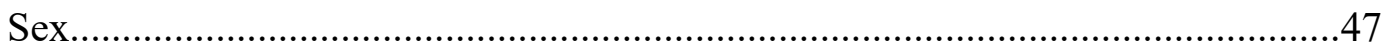

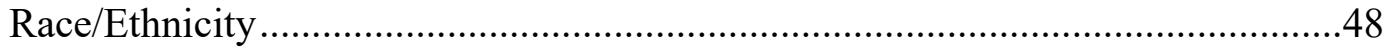

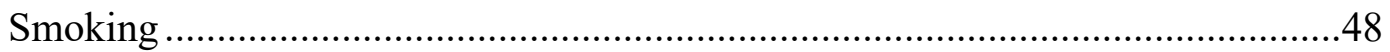

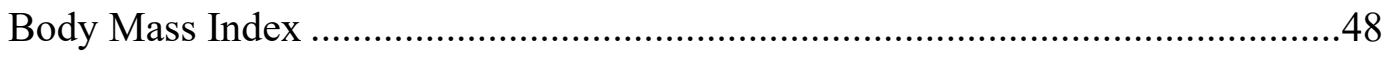

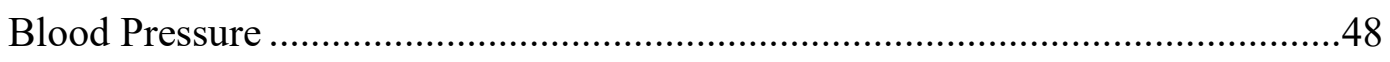

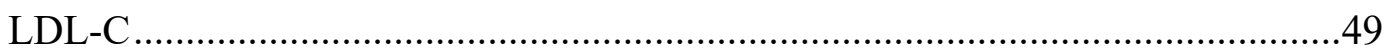

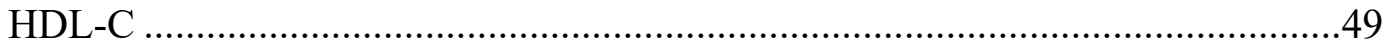

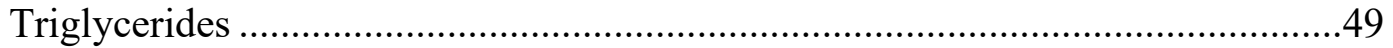

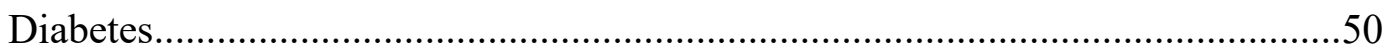

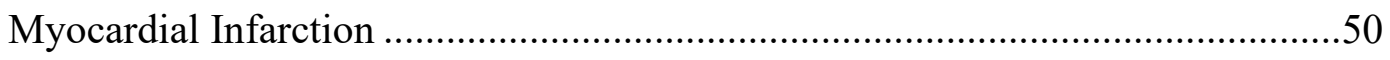

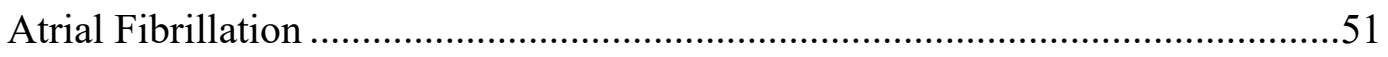

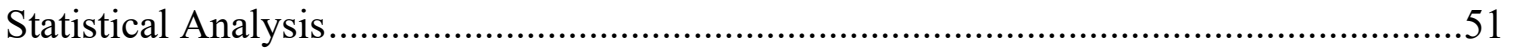

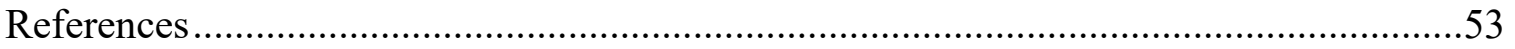

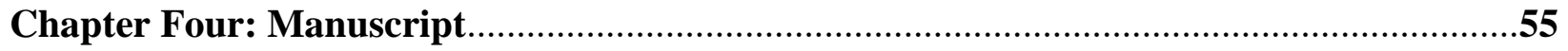

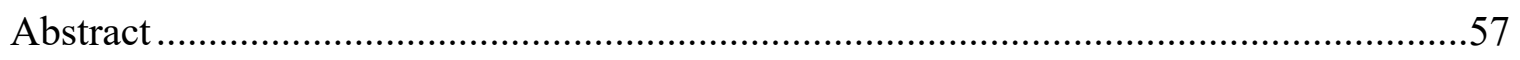

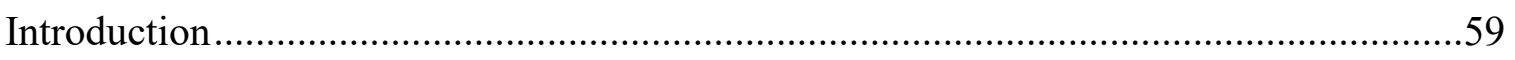

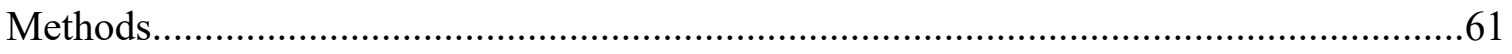

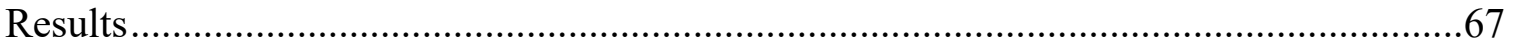

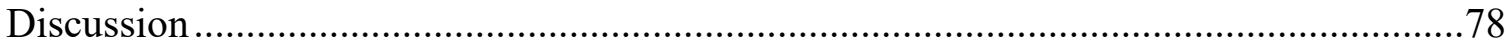

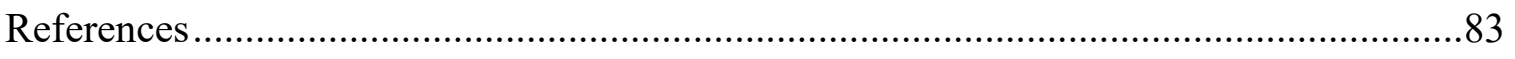

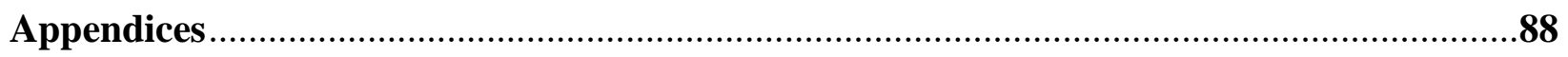

Appendix A: University of North Florida IRB Approval Letter ..................................89

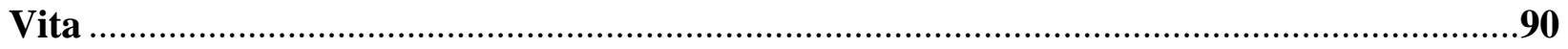




\section{List of Tables}

Tables

Page

\section{Chapter Two: Review of Literature}

1. Heart failure diagnostic criteria ........................................... 18

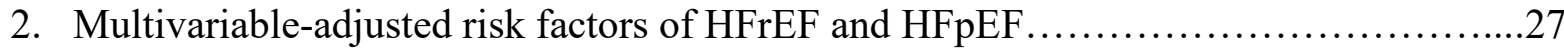

\section{Chapter Four: Manuscript}

1. Baseline characteristics of adults with and without heart failure by subtype.

2. Hazard ratios associated with total brachial artery reactivity $<7.9 \%$ and risk of incident heart failure .70

3. Hazard ratios associated with risk of incident heart failure in the parsimonious model.

4. Hazard ratios associated with total brachial artery reactivity $<7.9 \%$ and risk of incident heart failure with reduced ejection fraction .73

5. Hazard ratios associated with risk of incident heart failure with reduced ejection fraction in the parsimonious model. .74

6. Hazard ratios associated with total brachial artery reactivity $<7.9 \%$ and risk of incident heart failure with preserved ejection fraction. .76

7. Hazard ratios associated with risk of incident heart failure with preserved ejection fraction in the parsimonious model. 


\section{Nomenclature}

$\begin{array}{ll}\mathrm{Kg} / \mathrm{m}^{2} & \text { kilograms per meter squared } \\ \mathrm{mmHg} & \text { millimeters of mercury } \\ \mathrm{mg} / \mathrm{dL} & \text { milligrams per deciliter } \\ \mathrm{pg} / \mathrm{mL} & \text { picogram per liter }\end{array}$




\section{List of Abbreviations}

\begin{tabular}{|c|c|}
\hline AF & atrial fibrillation \\
\hline $\mathrm{ACCF}$ & American College of Cardiology Foundation \\
\hline AHA & American Heart Association \\
\hline ARIC & Atherosclerosis Risk in Communities \\
\hline BMI & body mass index \\
\hline $\mathrm{BP}$ & blood pressure \\
\hline $\mathrm{CI}$ & confidence interval \\
\hline CVD & cardiovascular disease \\
\hline CHD & coronary heart disease \\
\hline $\mathrm{CHF}$ & congestive heart failure \\
\hline DHF & diastolic heart failure \\
\hline $\mathrm{EF}$ & ejection fraction \\
\hline eNOS & endothelial nitric oxide synthase \\
\hline FHS & Framingham Heart Study \\
\hline FMD & flow-mediated dilation \\
\hline $\mathrm{HF}$ & heart failure \\
\hline $\mathrm{HFpEF}$ & heart failure with preserved ejection fraction \\
\hline HFrEF & heart failure with reduced ejection fraction \\
\hline HDL & high density lipoprotein \\
\hline HTN & hypertension \\
\hline $\mathrm{HR}$ & hazard ratio \\
\hline
\end{tabular}




\begin{tabular}{|c|c|}
\hline LDL & low density lipoprotein \\
\hline LV & left ventricle/left ventricular \\
\hline LVEF & left ventricular ejection fraction \\
\hline MESA & Multi-Ethnic Study of Atherosclerosis \\
\hline MI & myocardial infarction \\
\hline NCEP & National Cholesterol Education Program \\
\hline NDI & National Death Index \\
\hline NO & nitric oxide \\
\hline RAAS & renin-angiotensin-aldosterone system \\
\hline ROS & reactive oxygen species \\
\hline RV & right ventricle/right ventricular \\
\hline SAS & statistical analysis software \\
\hline SHF & systolic heart failure \\
\hline SNS & sympathetic nervous system \\
\hline TBAR & total brachial artery reactivity \\
\hline TTCHF & time to congestive heart failure \\
\hline WC & waist circumference \\
\hline
\end{tabular}


Purpose:

Methods:

Results: unknown or borderline EF (41-49\%). Crude analysis revealed that those with TBAR below the median have significantly higher risk of HF (HR 1.46; 95\% CI 1.13-1.88, $\mathrm{p}<0.01$ ) and HFrEF (HR 1.61; 95\% CI 1.07-2.43, $\mathrm{p}<0.05$ ). Following adjustment for known HF risk factors (e.g. age, gender, race, blood pressure, others), these relationships were no longer statistically 
significant. Borderline significant results were revealed in those with HFpEF (HR 1.43; 95\% CI 0.97-2.12, p=0.06). Kaplan-Meier curves suggest significantly lower risks of developing HF and HFrEF in those with TBAR above the median (log-rank $\mathrm{p} \leq 0.05$ for both). When examined as a continuous variable, with a cut point of $50 \%$ for EF, every 1-standard deviation (9.7\%) increase in TBAR resulted in a 19\% and $29 \%$ decrease in risk of $\mathrm{HF}(\mathrm{p}<0.05)$ and $\operatorname{HFrEF}(\mathrm{p}=0.05)$, respectively.

Conclusion: $\quad$ Lower TBAR values were associated with higher rates of incident HF and HFrEF, suggesting a possible role of endothelial dysfunction in $\mathrm{HF}$ pathogenesis. The impact of other known HF risk factors may mediate this relationship, thus further research is warranted. 
Chapter One: Introduction 
The American College of Cardiology Foundation (ACCF)/ American Heart Association (AHA) defines heart failure (HF) as "a complex clinical syndrome that results from any structural or functional impairment of ventricular filling or ejection of blood" (1). The abnormalities associated with this syndrome result in reduced cardiac output and/or elevated intracardiac pressures (2). Consequently, the heart is unable to pump blood to the body at a rate matching its needs. Based on systolic function measured by left ventricular ejection fraction (LVEF), HF can be classified into two major subtypes: heart failure with reduced ejection fraction (HFrEF), and heart failure with preserved ejection fraction (HFpEF). The two subtypes display marked differences in etiology, risk factors, and response to treatment (3-5).

It is estimated that $\mathrm{HF}$ affects 1 to $3 \%$ of the population worldwide and can increase to $10 \%$ or more in populations over 70 years old (4-6); the number of persons is projected to increase $46 \%$ by 2030 (7). According to statistics approximately one-half of the patients with HF have HFrEF and the other half have HFpEF $(7,8)$. Despite major advances in HF management and treatment, mortality remains high $(5,6)$.

Evidence suggest that endothelial dysfunction may be a phenotypic expression of HF and may play a role in its pathogenesis $(8-12)$. Total brachial artery reactivity (TBAR) is a novel noninvasive measurement that evaluates endothelial function using ultrasound imaging. This measurement uses a baseline brachial artery diameter following cuff deflation and compensates for the absence of a stereotactic device, restricting errors associated with ultrasound probe displacement (13). TBAR has been found to be an independent predictor of incident coronary heart disease (CHD) events.

This chapter includes pertinent background information relating to HF and TBAR. A focused review of the existing literature regarding the relationship between these variables is also 
included. The chapter concludes with the purpose and significance of the research, the project description, and limitations inherent to the study design.

\section{BACKGROUND}

\section{HEART FAILURE}

$\mathrm{HF}$ is considered a growing public health problem with a prevalence over 5.8 million adults in the United States (U.S) and approximately 37.7 million worldwide $(5,14)$. Even though these estimates vary depending on the study population and the diagnosis criteria, the prevalence of HF is approximately 1 to $3 \%$ and increases to $10 \%$ with advancing age $(4-6,14)$. The increased prevalence of HF over time may reflect a combination of growth of the aging population, changes in the prevalence of risk factors, and improvements in the recognition, management, and treatment of $\operatorname{HF}(14,15)$.

Annually in the U.S more than 550,000 individuals are diagnosed with incident HF, and it is estimated that 1 in 5 men and women over 40 years of age will develop HF during their lifetime $(14,16)$. According to the Framingham Heart Study (FHS), the incidence of HF is about 10 per 1,000 person-years in those $>65$ years of age, but it seems to be stabilizing, or even decreasing in women in which the incidence declined about 30\% between 1950-1969 and 1990-1999 (14-16). Data from the Atherosclerosis Risk in Communities (ARIC) showed the lowest HF incidence in white women (3.4 per 1,000 person-years) and the highest in black men (9.1 per 1,000 personyears) (14). However, these differences were attenuated after adjustment for risk factors, such as diabetes, and hypertension. 
Additionally, HF is considered a leading cause of hospitalization among the elderly, and one of the most frequent causes of urgent hospitalizations in the Medicare population $(5,17)$. Although efforts to find effective therapeutics have reduced the prevalence of rehospitalizations to $30-50 \%$, the mortality rates remain high $(2,5,6,14)$. According to statistics, the mortality rate in patients who are hospitalized is approximately $4 \%$ and increases to $10 \%$ within one-month of discharge (17). Based on the FHS, the 30-day, 1-year, and 5-year mortality, is nearly 10\%, 20$30 \%$, and $45-60 \%$, respectively (14-16). HF has extensive implications not only in terms of mortality and morbidity but also confers a considerable burden to the health-care system $(2,4,5$, 14, 18). The World Bank estimates that the global economic cost of managing HF is about $\$ 108$ billion annually (4) and in the U.S, it is expected to rise from $\$ 20.9$ billion in 2012 to $\$ 53.1$ billion by $2030(5)$.

The diagnosis of HF is challenging since it is based on an integration of the patient's history, evaluation of signs and symptoms, physical examination, imaging data, and laboratory findings, that require judgment and experience $(5,17)$. In 1971 the FHS established clinical criteria for HF diagnosis according to physical examination and physician adjudication (5). A diagnosis of definite HF required the combination of at least two major criterion (e.g., rales, S3 gallop, neckvein distension), or one major criterion and two minor criterion (e.g., ankle edema, dyspnea, etc.) (19). However, depending on the clinical and epidemiological studies, definitions and diagnostic criteria of HF vary widely and are prone to misclassification (5).

The HF entity has different characteristics depending on age, sex, race/ethnicity, LVEF, and HF etiology. Presentation of HF signs and symptoms differs widely and range from those with severe LV systolic dysfunction and low cardiac output to those with severe hypertension and normal or near-normal LV systolic function (4). As such, EF has been considered important in 
classification of patients with HF. Based on the ACCF/AHA guidelines (1) HFrEF, also referred to as systolic HF, is defined "as the clinical diagnosis of $\mathrm{HF}$ with an $\mathrm{EF} \leq 40 \%$ ", and $\mathrm{HFpEF}$, referred to as diastolic HF, "as HF with an EF $\geq 50 \%$ "; those with an EF between $40-49 \%$ are considered borderline.

Several studies have estimated that among individuals with HF, approximately half of the events are characterized by reduced EF and the other half by preserved EF $(1,17,20)$. The overall number of patients living with HF seems to be increasing over time, because of aging of the population, general population growth, and improved survival $(5,21)$. However, the prevalence of HFpEF is higher than that of HFrEF. According to data from the AHA, the proportion of patients hospitalized with HFpEF increased from 33\% in 2005 to 39\% in 2010 (5). The two subtypes exhibit marked differences in etiology, risk factors, and response to treatment $(3,20)$. Those who develop HFpEF tend to be older women, with a history of hypertension (HTN) or atrial fibrillation (AF), and those with HFrEF tend to be males, with a history of cardiomyopathy and myocardial infarction $(\mathrm{MI})(3,14)$. Despite the advances in treatments, strategies that have demonstrated to be effective among those with HFrEF are ineffective among those with $\operatorname{HFpEF}(1,14)$. For this reason, there has been an increased interest in identifying better diagnosis, phenotype characterization, and novel treatments between the two subtypes.

\section{TOTAL BRACHIAL ARTERY REACTIVITY}

The brachial artery responds to shear stress induced by increasing blood flow during reactive hyperemia in a similar way to the coronary arteries; as such it is commonly used as a surrogate for evaluating coronary endothelial function (22). The total brachial artery reactivity (TBAR) is a noninvasive measurement that evaluates endothelial function using ultrasound imaging. It relies on obtaining a baseline diameter of the artery following cuff deflation. After 5 
minutes of occlusion with a blood pressure cuff to induce forearm ischemia, TBAR is determined as the maximal brachial artery diameter compared to a baseline measurement (13). Although the baseline artery diameter is commonly measured before cuff inflation, this method might compensate for the absence of a stereotactic device, limiting the errors associated with ultrasound probe displacement (13). In a study comparing pre-occlusion, occlusion, and post-occlusion baseline brachial artery measurements, there were no significant differences between them; in addition, peak dilation values were not significantly different when calculated from pre-occlusion, occlusion or post-occlusion baseline diameters (23).

The expected response of a healthy endothelium is an increase in brachial artery diameter, secondary to the endogenous release of nitric oxide (NO) $(9,24-26)$. On the other hand, reduced bioavailability of NO and increased formation of reactive oxygen species (ROS) within the vascular wall are markers of endothelial dysfunction $(9,24,27)$. Previous studies have found a strong association between endothelial dysfunction measured by flow-mediated dilation (FMD), incident cardiovascular disease (CVD) events, and heart failure (12, 22, 25, 28, 29). Since attenuated vascular responses seem to occur prior to the development of atherosclerosis, the early assessment of endothelial function has become appealing to evaluate cardiovascular risk $(25,26$, 30).

In a study conducted by Polak et al. (13) using data from the Multi-Ethnic Study of Atherosclerosis (MESA), increased TBAR values were associated with a $22 \%$ lower risk of incident CHD events per SD increase (9.7 SD) (HR 0.78; 95\% CI 0.67-0.91, p = 0.001). In addition, they found that a TBAR above the median of $7.87 \%$ was associated with a $31 \%$ lower risk of CHD events (HR 0.69; 95\% CI 0.55-0.87, $\mathrm{p}=0.001)$. They concluded that TBAR is an independent predictor of first time CHD events. 


\section{FOCUSED LITERATURE REVIEW}

Evidence suggest that endothelial dysfunction may be a phenotypic expression of HF and may play a role in the pathogenesis of this entity $(8,9,12,31)$. It is possible that the characteristic neurohumoral activation in HF might be associated with an imbalance between NO and ROS (27). Increases in ROS result in decreased bioavailability of NO, negatively impacting the expression and activation of endothelial NO synthase (eNOS) and subsequent endothelial function $(6,26,32)$.

Although TBAR has been associated with increased risk of cardiovascular outcomes in a community-based study (13), limited information is currently available on the impact of TBAR on incident HF, HFrEF, and HFpEF.

\section{PURPOSE AND SIGNIFICANCE}

Despite the efforts to establish clear differences between HFrEF and HFpEF, the etiology, pathophysiology, and effective treatments for HFpEF are still unclear. Determining the relationship between endothelial dysfunction as a potential target and incident HF subtypes may help to elucidate a better understanding of the complex syndrome that is HF. Thus, the aim of this study was to investigate whether TBAR was associated with overall incident HF, HFrEF, and HFpEF in a diverse population-based sample of U.S. adults 45-84 years of age. The research questions addressed include:

1. Is there an association between TBAR and the cumulative risk of overall incident HF, and the two HF subtypes?

2. Is TBAR an independent predictor for HF and its subtypes?

3. Is there a significant difference between TBAR values for HFrEF and HFpEF? 
To the extent of our knowledge, this is the first study to examine the association between TBAR and risk of HF stratified by EF in a population-based sample of U.S. adults who participated in MESA.

\section{PROJECT DESCRIPTION}

The data for this study came from MESA (33), an ongoing, multicenter prospective cohort study of CVD, sponsored by the National Heart Lung and Blood Institute of the National Institutes of Health. The MESA study is a diverse, population-based sample of 6,814 men and women aged 45-84, with no history of clinical CVD at baseline. Cox proportional hazards regression models were used to calculate multivariable adjusted hazard ratios and $95 \%$ confidence intervals to determine the risk of HF overall, $\mathrm{HFrEF}$, and $\mathrm{HFpEF}$ according to TBAR values.

\section{LIMITATIONS}

1. The sample size was relatively small for those with HF, HFrEF and HFpEF, limiting the generalizability of the results.

2. TBAR and EF were measured at a single time point, therefore potential changes in these values over time were not included in the analysis.

3. Time to first HF event was used to define incident HF, resulting in the exclusion of subsequent HF events with potential changes in EF over time.

4. Those individuals without EF data at the time of their first HF event were included in the analysis of HF overall, but not when categorized by HF subtypes. 
5. TBAR was measured with an occlusion cuff placed proximal to the ultrasound probe (upper right forearm), which may indicate that the vasodilatory response is not mediated exclusively by NO.

6. Some of the covariates used for the analysis are subject to recall bias and self-report bias. 


\section{REFERENCES}

1. Yancy C, Jessup M, Bozkurt B, et al. 2013 ACCF/AHA guideline for the management of heart failure: Executive summary: A report of the American college of cardiology foundation/American heart association task force on practice guidelines. Circulation. 2013;128(16):1810-1852.

2. Tomasoni D, Adamo M, Lombardi CM, Metra M. Highlights in heart failure. ESC Heart Fail. 2019;6:1105-1127.

3. Lee MP, Glynn RJ, Schneeweiss S, et al. Risk factors for heart failure with preserved or reduced ejection fraction among Medicare beneficiaries: Applications of competing risks analysis and gradient boosted model. Clin Epidemiol. 2019; 12:607-616.

4. Kurmani S, Squire I. Acute heart failure: definition, classification and epidemiology. Curr Heart Fail Rep. 2017;14(5):385-392.

5. Ziaeian B, Fonarow GC. Epidemiology and aetiology of heart failure. Nat Rev Cardiol. 2016; 13(6):368-378.

6. Giannitsi S, Mpougiaklh M, Bechlioulis A, Naka K. Endothelial dysfunction and heart failure: A review of the existing bibliography with emphasis on flow mediated dilation. JRSM Cardiovasc Dis. 2019; 8:1-7.

7. Parikh KS, Sharma K, Fiuzat M, et al. Heart failure with preserved ejection fraction expert panel report. JACC Heart fail. 2018; 6(8):619-632.

8. Virani SS, Alonso A, Benjamin EJ, et al. Heart disease and stroke statistics-2020 update: a report from the American Heart Association. Circulation. 2020; 9(141): e139-e596. 
9. Chong AY, Blann AD, Lip GYH. Assessment of endothelial damage and dysfunction: observations in relation to heart failure. $Q J$ Med. 2003; 96:253-267.

10. Heitzer T, Baldus S, Von Kodolitsch Y, et al. Systemic endothelial dysfunction as an early predictor of adverse outcome in heart failure. Arterioscler Thromb Vasc Biol. 2005; 25(6):1174-1179.

11. Alem MM. Endothelial dysfunction in chronic heart failure: assessment, findings, significance, and potential therapeutic targets. Int. J. Mol. Sci. 2019; 20(13):3198.

12. Ärnlöv J, Sang Y, Ballew SH, et al. Endothelial dysfunction and the risk of heart failure in a community-based study: the Multi-Ethnic Study of Atherosclerosis. ESC Heart Fail. 2020; 7(6):4231-4240.

13. Polak J, Ouyang P, Vaidya D. Total brachial artery reactivity and first time incident coronary heart disease events in a longitudinal cohort study: The multi-ethnic study of atherosclerosis. PLoS ONE. 2019; 14(4): e0211726.

14. Bui AL, Horwich TB, Fonarow GC. Epidemiology and risk profile of heart failure. Nat Rev Cardiol. 2011; 8(1):30-41.

15. Levy D, Kenchaiah S, Larson MG, et al. Long-term trends in the incidence of and survival with heart failure. N Engl J Med. 2002; 347(18):1397-402.

16. Lloyd-Jones DM, Larson MG, Leip EP, et al. Lifetime risk for developing congestive heart failure: the Framingham Heart Study. Circulation. 2002; 106(24):3068-72.

17. Pfeffer MA, Shah AM, Borlaug BA. Heart failure with preserved ejection fraction in perspective. Circ Res. 2019; 124(11):1598-1617.

18. Roger VL. Epidemiology of heart failure. Circ Res. 2013; 113(6): 646-659. 
19. Mahmood SS, Wang TJ. The epidemiology of congestive heart failure: the Framingham Heart Study perspective. Glob Heart. 2013;1(8):77-82.

20. Dunlay SM, Weston SA, Jacobsen SJ, et al. Risk factors for heart failure: a populationbased case-control study. Am J Med. 2009; 122(11):1023-1028.

21. Groenewegen A, Rutten FH, Mosterd A, Hoes AW. Epidemiology of heart failure. Eur. J. Heart Fail. 2020; 22(8):1342-1356.

22. Inaba Y, Chen JA, Bergmann SR. Prediction of future cardiovascular outcomes by flowmediated vasodilatation of brachial artery: A meta-analysis. Int J Cardiovasc Imaging. 2010; 26(6):631-640.

23. Ostrem JD, Dengel DR, Marlatt KL, et al. Comparison of baseline brachial artery measurements and effect on peak flow-mediated dilation. Clin Physiol Funct Imaging. $2015 ; 35(1): 34-40$.

24. Green DJ, Jones H, Thijssen D, et al. Flow-mediated dilation and cardiovascular event prediction. Does nitric oxide matter? Hypertension. 2011; 57(3):363-369.

25. Ras RT, Streppel MT, Draijer R, et al. Flow-mediated dilation and cardiovascular risk prediction: A systematic review with meta-analysis. Int J Cardiol. 2012; 168:344-351.

26. Areas GPT, Mazzuco A, Caruso FR, et al. Flow-mediated dilation and heart failure: a review with implications to physical rehabilitation. Heart Fail Rev. 2019; 24(1):69-80.

27. Bauersachs J, Widder JD. Endothelial dysfunction in heart failure. Pharmacol Rep. 2008. 60(1):119-126. 
28. Shechter M, Issachar A, Marai I, et al. Long-term association of brachial artery flowmediated vasodilation and cardiovascular events in middle-aged subjects with no apparent heart disease. Int J Cardiol. 2009; 134(1):52-58.

29. Yeboah J, Folsom AR, Burke GL, et al. Predictive value of brachial flow-mediated dilation for incident cardiovascular events in a population-based study: The multi-ethnic study of atherosclerosis. Circulation. 2009; 120(6):502-509.

30. Hashimoto M, Kozaki K, Eto M, et al. Association of coronary risk factors and endothelium-dependent flow-mediated dilatation of the brachial artery. Hypertens Res. $2000 ; 23(3): 233-238$.

31. Kishimoto S, Kajikawa M, Maruhashi T, et al. Endothelial dysfunction and abnormal vascular structure are simultaneously present in patients with heart failure with preserved ejection fraction. Int J Cardiol. 2016; 231:181-7.

32. Widmer R.J, Lerman A. Endothelial dysfunction and cardiovascular disease. Glob Cardiol Sci Pract. 2014; 2014(3):291-308.

33. Bild DE, Bluemke DA, Burke GL, et al. Multi-Ethnic Study of Atherosclerosis: objectives and design. Am J Epidemiol. 2002; 156(9):871-881. 
Chapter Two: Review of Literature 
According to the American Heart Association (AHA) heart failure (HF) is a "complex clinical syndrome that results from any structural or functional impairment of ventricular filling or ejection of blood" (1). As a result, the heart is incapable of providing sufficient blood flow to meet metabolic demands or to accommodate systemic venous return $(2,3)$. Although HF results from injury to the myocardium, this syndrome is characterized by multisystemic abnormalities, and a complex pattern of neurohumoral changes (4-6). Multiple etiologies have been associated with HF including ischemic heart disease, hypertension, diabetes, and other less common causes such as cardiomyopathies, valvular disease, infections, and arrhythmias (2). As heart function decreases, patients with HF experience numerous symptoms, which include dyspnea from pulmonary congestion, fatigue, poor exercise tolerance, fluid retention, and edema from impaired venous return $(2,7)$.

In HF the underlying injury of functional myocardial cells can result in right ventricular (RV) dysfunction, left ventricular (LV) dysfunction, or both. Pure right-side HF is uncommon, and in fact, the most common cause of RV dysfunction is LV dysfunction (2). As the RV fails, the resultant accumulation of blood in the ventricle leads to elevated right atrial pressure and increased pressure in the vena cava (superior and inferior) which impairs venous return. Left-side HF can be classified into two categories based on left ventricular ejection fraction (LVEF): systolic and diastolic $(2,8)$. Although different etiologies of HF tend to affect more systolic or diastolic function, most patients with systolic dysfunction also have a component of diastolic dysfunction.

Ejection fraction (EF) refers to the ratio of the amount of blood ejected from the ventricle in one heartbeat to the ventricular-end diastolic volume. According with the most recent guidelines by the American College of Cardiology Foundation (ACCF)/(AHA), HF with reduced EF (HFrEF), also referred to as systolic HF, is defined as an $\mathrm{EF} \leq 40 \%$, whereas $\mathrm{HF}$ with preserved 
$\mathrm{EF}$ (HFpEF), also referred to as diastolic $\mathrm{HF}$, is defined as an $\mathrm{EF} \geq 50 \%$. Those with an $\mathrm{EF}$ between $41-49 \%$ are considered to have HF with mid-range EF (HFmrEF) $(1,9)$. These subtypes display marked differences in etiology, risk factors, and response to treatment $(7,10,11)$.

This chapter includes a focused literature review on the terminology of HF, HFrEF, and HFpEF, diagnosis, pathophysiology, and known risk factors associated with each HF subtype. Existing literature that has investigated the relationship between endothelial function and HF overall, HFrEF and HFpEF are also included. It concludes with a summary of the literature and the explanation of the need for additional research.

\section{TERMINOLOGY}

Over the past 20-30 years the classification terminology for HF has evolved considerably, however, disagreements and uncertainties remain (7). The term HF has been used to refer to those patients with established chronic heart failure and is limited to stages at which clinical symptoms are present. During the course of this entity, episodes of worsening symptoms and signs can occur, resulting in a need for urgent care that may require hospitalization. The term used to define these exacerbations is acute decompensated $\operatorname{HF}(12,13)$. Congestive heart failure is another term that is frequently used interchangeably with HF. However, because some patients do not exhibit signs or symptoms of volume overload, the term HF is preferred over congestive heart failure $(1,14)$. Depending on the patient's disease stage, these terms can be applied to the same patient at different times (9). It is important to note that HF is not synonymous with cardiomyopathy, since the latter corresponds to a group of diseases of the myocardium that frequently progress to HF. In that sense 
cardiomyopathy describes structural or functional reasons associated with the development of HF $(1,7,9)$.

Previously, the terms systolic and diastolic dysfunction regardless of EF were used to classify HF. However, because abnormalities of systolic and diastolic function coexist in most patients, these terms are no longer appropriate $(1,9,13)$. The preferred terminology used today is HF with reduced EF (HFrEF), and HF with preserved EF (HFpEF) based on LVEF, assessed by echocardiography. Since patients with HF display a wide variety of signs and symptoms, that range from severe LV systolic dysfunction and low cardiac output (CO) to severe hypertension and normal or near-normal LV systolic function, classification based on EF is recommended (11). The importance of using LVEF as a means to classify HF allows differentiation of underlying etiologies, demographics, comorbidities, and response to therapies between $\operatorname{HF}$ subtypes $(1,9,13)$.

\section{DIAGNOSIS}

Since HF is defined as a clinical syndrome with many etiologies and not a disease, its diagnosis relies on a careful patient history and clinical examination $(1,9,12)$. Multiple criteria have been proposed to diagnose HF in epidemiological studies, however, there is not a validated gold standard (15). These include Framingham (16), Boston (17), Gothenburg (18), and the European Society of Cardiology criteria (19), all of which rely on a combination of data from the medical history, physical examination, and radiographic evidence $(12,14)$. A description of these four criteria is listed in Table 1 below. 


\begin{tabular}{|c|c|c|c|}
\hline Framingham & Boston & Gothenburg & $\begin{array}{c}\text { European Society of } \\
\text { Cardiology }\end{array}$ \\
\hline $\begin{array}{l}\text { Major Criteria } \\
\text {-Paroxysmal nocturnal } \\
\text { dyspnea or orthopnea } \\
\text {-Neck vein distension } \\
\text {-Rales } \\
\text {-Cardiomegaly } \\
\text {-Acute pulmonary } \\
\text { edema } \\
\text {-S3 gallop } \\
\text {-Increased venous } \\
\text { pressure } \geq 16 \mathrm{~cm} \text { water } \\
\text {-Circulation time } \geq 25 \\
\text { seconds } \\
\text {-Hepatojugular reflux } \\
\text { Minor Criteria } \\
\text {-Ankle edema } \\
\text {-Night cough } \\
\text {-Dyspnea on exertion } \\
\text {-Hepatomegaly } \\
\text {-Pleural effusion } \\
\text {-Vital capacity } \\
\text { decreased } 1 / 3 \text { from } \\
\text { maximum } \\
\text {-Tachycardia rate of } \\
\geq 120 / \text { min) } \\
\text { Major or Minor } \\
\text {-Writerion } \\
\text { days in response to } \\
\text { treatment } \\
\end{array}$ & $\begin{array}{l}\text { Category I: History } \\
\text {-Rest dyspnea (4pts) } \\
\text {-Orthopnea (4pts) } \\
\text {-Paroxysmal nocturnal } \\
\text { dyspnea } \\
\text { (3 pts) } \\
\text {-Dyspnea on walking on } \\
\text { level (2pts) } \\
\text {-Dyspnea on climbing } \\
\text { (1pt) } \\
\text { Category II: Physical } \\
\text { examination } \\
\text {-Heart rate abnormality } \\
\text { (1-2pts) } \\
\text {-Jugular venous pressure } \\
\text { elevation } \\
\text { (1-2 pts) } \\
\text {-Lung crackles } \\
\text { (1-2pts) } \\
\text {-Wheezing ( } 3 \text { pts) } \\
\text {-Third heart sound } \\
\text { (3 pts) } \\
\text { Category III: Chest } \\
\text { radiography } \\
\text {-Alveolar pulmonary } \\
\text { edema (4 pts) } \\
\text {-Interstitial pulmonary } \\
\text { edema ( } 3 \text { pts) } \\
\text {-Bilateral pleural } \\
\text { effusions ( } 3 \text { pts) } \\
\text {-Cardiothoracic } \\
\text { ratio } \geq 0.50 \text { ( } 3 \text { pts) } \\
\text {-Upper-zone flow } \\
\text { redistribution (2 pts) } \\
\text { HEART FAILURE } \\
\text { Definite } 8-12 \text { pts, } \\
\text { possible 5-7pts, unlikely } 4 \\
\text { pts or less }\end{array}$ & $\begin{array}{l}\text { Cardiac Score } \\
\text {-History of heart disease } \\
(1-2 \mathrm{pts}) \\
\text {-Angina (1-2pts) } \\
\text {-Edema (1pt) } \\
\text {-Nocturnal Dyspnea (1pt) } \\
\text {-Rales (1pt) } \\
\text { Atrial fibrillation (1pt) } \\
\text { Pulmonary Score } \\
\text {-History of Chronic } \\
\text { bronchitis/asthma } \\
\text { (1-2pts) } \\
\text {-Cough, phlegm, or } \\
\text { wheezing (1pt) } \\
\text {-Rhonchi (2pts) } \\
\text { Cardiac and pulmonary } \\
\text { score are calculated and } \\
\text { used to differentiate } \\
\text { cardiac from pulmonary } \\
\text { dyspnea }\end{array}$ & $\begin{array}{l}\text { 1. Symptoms of } \\
\text { heart failure (at } \\
\text { rest or during } \\
\text { exercise) and } \\
\text { 2. Objective } \\
\text { evidence of } \\
\text { cardiac } \\
\text { dysfunction (at } \\
\text { rest) and } \\
\text { 3. Response to } \\
\text { treatment } \\
\text { directed } \\
\text { towards heart } \\
\text { failure (in cases in which } \\
\text { diagnosis } \\
\text { is in doubt). } \\
\text { Criteria } 1 \text { and } 2 \\
\text { should be fulfilled in all } \\
\text { cases. }\end{array}$ \\
\hline
\end{tabular}

Note. Adapted from "Epidemiology of heart failure" by Roger. Circulation Research. 2013; 113(6): 646-659. 
Mosterd et al. (18) compared six different HF scores, including the Framingham and the Boston criteria. They found that five out of the six scores (excluding the Men Born in 1913) were similar in the recognition of HF with a high sensitivity for the detection of definite HF. However, they concluded that objective measurements of cardiac function might be necessary to accurately detect early stages of HF. Evidence of an underlying cardiac cause is crucial to the diagnosis of HF since identification of the specific pathology determines the treatment used (9).

Although these criteria identify HF, they do not differentiate between HF subtypes. The latest guidelines have suggested the use of LVEF, usually measured using echocardiography, radionuclide technique, or cardiac magnetic resonance, to classify HF patients as HFrEF or HFpEF. The 2013 ACCF/AHA guidelines to define HF subtypes uses a LVEF of $\leq 40 \%$ to define HFrEF and a LVEF of $\geq 50 \%$ to define HFpEF (1). Those patients with a LVEF in the range of $41-$ 49\% are now defined as HFmrEF, and present a combination of mild systolic dysfunction, with elements of diastolic dysfunction.

Because the diagnosis of HFpEF is largely based on exclusion of noncardiac causes of symptoms suggestive of HF, patients with HFpEF frequently experience delayed diagnosis and limited treatment options $(7,9)$. The 2016 European Society of Cardiology guidelines established specific criteria to define HFpEF, including:

1. The presence of symptoms and/or signs of HF.

2. Evidence of preserved or normal LVEF ( $\geq 50 \%$ or $40-49 \%$ for HFmrEF).

3. Elevated levels of natriuretic peptides (NPs) (B-type NP $>35 \mathrm{pg} / \mathrm{mL}$ and/or N-terminal pro$\mathrm{BNP}>125 \mathrm{pg} / \mathrm{mL})$.

4. Objective evidence of other cardiac functional and structural alterations underlying HF. 


\section{PATHOPHYSIOLOGY}

HF is caused by a structural and/or functional cardiac abnormality, that results in a reduced $\mathrm{CO}$ and/or elevated intracardiac pressures (9). The etiology of HF is diverse and sometimes there is overlap between possible categories. Among the most common causes are ischemic heart disease, hypertension, and diabetes (2). Although in HF there is usually a myocardial abnormality that causes systolic and/or diastolic ventricular dysfunction, abnormalities of the valves, pericardium, endocardium, heart rhythm and conduction are not uncommon. Other causes of HF include cardiomyopathies, infections (e.g., viral myocarditis), toxins (e.g., alcohol), and arrhythmias (9).

Depending on the etiology, some are more likely to affect systolic or diastolic function. However, most patients with systolic dysfunction also have a component of diastolic dysfunction (2). The impairment in LV function, results in a reduction of $\mathrm{CO}$ and mean arterial pressure (MAP) which leads to decreased tissue perfusion $(2,4)$. In addition, the increased amount of blood in the ventricle results in increased end-systolic and end-diastolic volumes. Consequently, there is an elevation of atrial pressure and subsequent elevated pressure in the lungs that forces fluid out of the pulmonary capillaries. The resultant pulmonary congestion is responsible for the clinical symptom of dyspnea (2).

RV dysfunction is usually secondary to LV dysfunction. Failure of the RV results in increased pressure in the vena cava which impairs venous return and subsequent peripheral edema. These conditions lead to activation of several compensatory mechanisms including the FrankStarling mechanism, neurohumoral activation, and ventricular remodeling $(2,4)$. Regardless of the beneficial effects of these mechanisms in early phases of this syndrome, they also play a detrimental role in the development and subsequent progression of HF. 
The Frank-Starling mechanism causes an increase in $\mathrm{CO}$ to maintain adequate tissue perfusion. The increased end-diastolic volume causes a stretch in the myocardium that results in greater force of contraction and subsequent greater $\mathrm{CO}$. However, over time the failing heart is unable to contract, and this compensatory mechanism is diminished, leading to pulmonary congestion (2). The second mechanism involves neurohumoral activation to maintain MAP. The decrease in MAP that is characteristic of HF, leads to the stimulation of the sympathetic nervous system (SNS) and the release of catecholamines (4). Activation of the SNS results in increased inotropy, chronotropy, and total peripheral resistance. Additionally, there is an activation of the renin-angiotensin-aldosterone system (RAAS), that leads to increased concentrations of renin, plasma angiotensin II, and aldosterone. Angiotensin II is a potent vasoconstrictor that has important effects on cardiac myocytes and may contribute to the endothelial dysfunction present in HF (2). Aldosterone is responsible for sodium and water retention to increase blood volume. Nonetheless, overstimulation by the SNS is associated with myocardial toxicity, ventricular remodeling, myocyte apoptosis and hypertrophy (4).

Ventricular remodeling refers to the alterations in the size, shape, structure, and function of the ventricle, because of chronic stress on the heart (2). Initially, these changes are compensatory to increase ventricular volume. In addition, ventricular mass and myocardial wall thickness increase to improve contractility. This remodeling process in HF is progressive leading to fibrosis, myocardial apoptosis, and contractile impairments.

\section{HFrEF}

The pathophysiology of HFrEF is well-understood and most of the therapeutic interventions available have proven effective for this HF subtype. The patterns of ventricular remodeling in HFrEF involve LV eccentric hypertrophy, LV chamber dilation, and markedly 
reduced end-systolic elastance $(10,20)$. HFrEF typically develops in response to an accelerated and a large-scale myocyte loss/dysfunction secondary to conditions such as acute myocardial infarction (MI), genetic abnormalities, myocarditis, or toxin effects (20).

Patients who develop HFrEF are more likely to be male with a history of coronary artery disease (CAD) and have a greater risk of dying from cardiovascular causes. The reduced systolic function characteristic in these patients result in low $\mathrm{CO}$ that is unable to meet the metabolic demands. Consequently, symptoms of fatigue and exercise intolerance become evident. Another direct consequence of the reduced contractility is an increase in end-diastolic pressure. Then is transferred to the pulmonary, portal, and peripheral circulation, resulting in extravasation of fluid and subsequent edema (21).

\section{HFPEF}

The pathophysiology of HFpEF seems to be associated with an underlying proinflammatory state that triggers a cascade of events causing increased cardiomyocyte stiffness and increased ventricular wall stiffness (22). Several risk factors interact in a complex manner that result in the presence of clinical symptoms such as dyspnea and fatigue on activity (14). HFpEF involves abnormalities in left ventricular relaxation, with reduced ventricular compliance, leading to the impairment of diastolic ventricular filling. Abnormalities that affect ventricular filling include an increase in myocardial stiffness of the myocytes and extracellular collagen matrix, infiltrative disorders, and abnormalities in the cardiac and systemic microvasculature. As a result, it requires elevated filling pressures to obtain normal LV end-diastolic volumes $(14,15,21)$. Common causes include CAD, hypertension, hypertrophic cardiomyopathy, obesity, diabetes, and chronic obstructive pulmonary disease $(10,14)$. 
HFpEF is characterized by concentric remodeling and LV hypertrophy, with small to normal cavity volumes $(14,15,20)$. Patients who develop HFpEF are more likely to be older, female, and have a history of hypertension or atrial fibrillation (AF). HFpEF represents over half of prevalent HF, however, regardless of the improvements in understanding the pathophysiology of this entity, effective therapies are yet to be stablished $(1,14,15,22)$. A possible explanation is that HFpEF is a heterogeneous condition consisting of several pathophysiological subtypes (23).

\section{SUMMARY}

Although HFrEF and HFpEF share similar signs, symptoms, and outcomes, they represent two different disorders, with individual pathogenesis pathways. As such, they should be studied and treated separately. In HFrEF, the usually patterns of ventricular remodeling involve LV eccentric hypertrophy and LV chamber dilation whereas in HFpEF concentric remodeling and LV hypertrophy with small to normal cavity volumes are common. However, it is important to note that neither remodeling pattern is unique to just HFrEF or HFpEF. For example, concentric hypertrophy has been also reported in a subset of patients with HFrEF. While HFrEF is associated with a direct myocyte loss/dysfunction, $\mathrm{HFpEF}$ is associated with an underlying proinflammatory state secondary to comorbid conditions. Identification of the underlying causes and pathophysiology of HF subtypes is critical for therapeutic reasons, as the precise pathology determines the specific treatment used.

\section{RISK FACTORS}

Several epidemiologic studies have examined the relationship between potential risk factors and HF overall, HFrEF, and $\operatorname{HFpEF}(10,24-27)$. Bui et al. (15) established major and minor 
clinical risk factors for the development of HF. Among the major risk factors are age, male sex, hypertension, LV hypertrophy, MI, valvular heart disease, obesity, and diabetes. Some of the minor risk factors include smoking, dyslipidemia, chronic kidney disease, albuminuria, sedentary lifestyle, and low socioeconomic status. Evidence suggests that identification and modification of some of these potential risk factors may prevent or delay the development of HF (9).

McKee et al. (16) were among the first to report the influence of age and gender in HF occurrence. They found that incidence rates of HF increased markedly with age and were higher in men than in women at all ages. Recent reports estimate that by the age of 40, people in the U.S have a one in five chance of developing HF and that it is more common in males until the age of $65(9,15)$

African Americans have the highest prevalence of HF at 4.6 per 1000 person-years, mostly related with the greater burden of atherosclerotic risk factors in this population. The prevalence of HF in Hispanic, Caucasian, and Asian Americans is 3.5, 2.4, and 1.0 per 1000 person-years, respectively (2). However, among a diverse sample of 39,578 participants from the Chicago Heart Association Detection Project in Industry, the Atherosclerosis Risk in Communities (ARIC) study, and the Cardiovascular Health Study, the lifetime risk for developing HF at age 45 years was 30 to $42 \%$ in white men, 20 to $29 \%$ in black men, 32 to $39 \%$ in white women, and 24 to $46 \%$ in black women (12).

Hypertension is a significant risk factor for HF overall (28). About $75 \%$ of all HF patients have preexisting hypertension and this risk factor alone doubles the risk of developing HF compared to normotensive patients (2). Dunlay et al. (26) reported the frequency of risk factors among 962 incident HF cases in Olmsted County. Hypertension was the most common (66\%) and accounted for $20 \%$ of HF cases in this population, with the greatest importance in women. Because 
of the high prevalence of hypertension, there is a great contribution to the population burden of HF. The greater the elevation in blood pressure, the greater the risk of developing HF (15).

Growing evidence illustrates a causal link between diabetes and obesity with HF independently of CAD and hypertension. Diabetes increases the risk of HF by approximately twofold in men and up to five-fold in women. The presence of obesity results in a two-fold higher risk of $\mathrm{HF}$ after adjustment for related risk factors $(13,28)$.

The presence of CAD markedly increases the risk of developing HF and correspond to the underlying etiology in up to 60 to $70 \%$ of patients with systolic dysfunction (28). He at al. (25) studied 13,643 subjects from the National Health and Nutrition Examination Survey (NHANES) and reported a relative risk for HF of 8.11 (95\% confidence interval (CI) 6.95-9.46) among those with CAD. Additionally, it is estimated that $36 \%$ of patients with prior MI will experience HF in the subsequent $7-8$ years $(13,15)$.

Smoking, low physical activity, and alcohol intake have also been linked to the development of HF. In the Coronary Artery Surgery Study, smoking was associated with a 47\% increased risk of developing HF (15). Also, an inverse relationship between physical activity and the risk of HF has been reported. Among subjects from NHANES, there was a 23\% higher risk of developing HF in those with low physical activity levels (25). Alcohol intake has a U-shaped relationship with the risk of developing HF; greater amounts of alcohol intake have been associated with the development of toxic cardiomyopathy (9).

New predictors of HF have been investigated including circulating biomarkers. In 2004, Wang et al. (29) showed that levels of plasma NPs predicted future HF among 3,346 individuals. 
Those with B-type NP concentrations in the top quintile ( $>20 \mathrm{pg} / \mathrm{mL}$ in men, and $>23.3 \mathrm{pg} / \mathrm{mL}$ in women) had a three-fold increased risk of developing HF over five years (30).

Lloyd et al. (31) determined the lifetime risk for developing HF among Framingham Heart Study (FHS) participants. At age 40 years, the lifetime risk for HF was $21.0 \%$ (95\% CI $18.7 \%$ to $23.2 \%)$ for men and $20.3 \%$ (95\% CI $18.2 \%$ to $22.5 \%)$ for women. For those in which HF occurred in the absence of MI, the lifetime risk was 1 in 9 for men and 1 in 6 for women, respectively.

Mosterd et al. (13) compared different risk factors for the occurrence of HF from three population-based studies, the FHS, the Cardiovascular Health Study, and the Rotterdam Study. Among the risk factors included were hypertension, MI, angina pectoris, diabetes, LV hypertrophy, valvular disease, AF, and chronic obstructive pulmonary disease (COPD). In the FHS, the population attributable risk (PAR) of hypertension was 39\% and 59\% for men and women, respectively. In the Cardiovascular Health Study, researchers found more than two-fold increased risk of HF in those with LV hypertrophy with a PAR of 6\%. In the Rotterdam Study, the highest relative risk was associated with diabetes for men, and COPD for woman. In addition, they reported that hypertensive women were 2.6 times more likely to develop HF that normotensive women, but this was not seen in men (15).

When examining risk factors by HF subtype, Ho et al. (32) studied differences in clinical predictors between $\mathrm{HFrEF}(\mathrm{EF} \leq 45 \%)$ and $\mathrm{HFpEF}$ ( $\mathrm{EF}>45 \%$ ) among participants from the FHS (Table 2). They reported as independent predictors for HFrEF male sex, hypertension, higher heart rate, prior cardiovascular disease (CVD), LV hypertrophy, and left bundle branch block (LBBB). On the other hand, higher body mass index, smoking, and AF were independent predictors for HFpEF. 


\begin{tabular}{|c|c|c|c|c|c|c|}
\hline & \multicolumn{3}{|c|}{ HFrEF } & \multicolumn{3}{|c|}{ HFpEF } \\
\hline & HR & $95 \%$ CI & $p$ value & HR & 95\% CI & p value \\
\hline & \multicolumn{6}{|c|}{ Demographics } \\
\hline Age, per year & 1.08 & $(1.06-1.09)$ & $<0.001$ & 1.13 & $(1.11-1.14)$ & $<0.001$ \\
\hline Female & 0.48 & $(0.37-0.63)$ & $<0.001$ & 1.14 & $(0.85-1.53)$ & 0.4 \\
\hline \multicolumn{7}{|l|}{ Clinical } \\
\hline Hypertension & 1.76 & $(1.28-2.41)$ & $<0.001$ & & & \\
\hline Heart rate, per $12 \mathrm{bpm}$ & 1.32 & $(1.19-1.48)$ & $<0.001$ & & & \\
\hline Prior myocardial infarction & 3.49 & $(2.48-4.90)$ & $<0.001$ & & & \\
\hline Prior coronary heart disease & 1.73 & $(1.27-2.34)$ & $<0.001$ & & & \\
\hline Cerebrovascular disease & 1.96 & $(1.34-2.86)$ & $<0.001$ & & & \\
\hline Diabetes mellitus & 2.91 & $(2.21-3.85)$ & $<0.001$ & 2.88 & $(2.05-4.05)$ & $<0.001$ \\
\hline BMI, per $4.7 \mathrm{~kg} / \mathrm{m}^{2}$ & & & & 1.41 & $(1.23-1.61)$ & $<0.001$ \\
\hline Current smoker & & & & 2.04 & $(1.39-2.99)$ & $<0.001$ \\
\hline Valvular disease & 2.44 & $(1.48-4.04)$ & $<0.001$ & 4.88 & $(3.05-7.82)$ & $<0.001$ \\
\hline \multicolumn{7}{|l|}{ ECG criteria } \\
\hline Atrial fibrillation & & & & 2.47 & $(1.57-3.89)$ & $<0.001$ \\
\hline Left ventricular hypertrophy & 2.73 & $(2.04-3.65)$ & $<0.001$ & & & \\
\hline Left bundle branch block & 3.41 & $(1.78-6.52)$ & $<0.001$ & & & \\
\hline
\end{tabular}

Note. Adapted from "Predictors of new-onset heart failure: differences in preserved versus reduced ejection fraction" by Ho et al. Circ Heart Fail. 2013; 6(2): 279-286.

Similarly, Lee et al. (33) reported that risk factors associated with an increased odds of HFrEF were male sex, prior MI, and LBBB. Among risk factors associated with HFpEF were female sex, elevated systolic blood pressure, and AF. Risk factors such as diabetes, smoking, and hypertension, were associated with both HF subtypes.

Using Medicare data from individuals $\geq 65$ years of age without HF, Lee at al. (10) evaluated the relationship between different risk factors and HF subtypes, and the relative influence (RI) of each predictor in the development of HFrEF and HFpEF. Male sex (hazard ratio 
(HR) 2.07, 95\% CI 1.81-2.37), cardiomyopathy (HR 4.37, 95\% CI 3.21-5.97), and MI (HR 1.94, 95\% CI 1.23-3.07) were strongly associated with HFrEF. Contrarily, pulmonary hypertension (HR 1.66, 95\% CI 1.23-2.22) and AF (HR 2.02, 95\% CI 1.80-2.26) were strongly associated with HFpEF. Cardiomyopathy and AF had the highest RI for the development of HFrEF (20.7\%) and HFpEF (8.4\%), respectively. Age and diabetes were associated with both HF subtypes, with age being the strongest predictor with a RI $>35 \%$.

Further research of the interaction between risk factors and the development of HF subtypes, will not only improve the diagnosis and treatment of individuals with HF, but also allow prevention strategies to be more effective in reducing the burden of HF (15).

\section{HEART FAILURE AND ENDOTHELIAL DYSFUNCTION}

Endothelial dysfunction has received increasing attention and a considerable amount of studies have been carried out to investigate its association with HF. Evidence indicates that endothelial dysfunction may play a role in the pathogenesis and progression of $\operatorname{HF}(6,34,35)$. The endothelium plays important physiological roles that involve regulation of blood vessel tone, permeability, metabolism, and hemostasis. Abnormal endothelial function has been associated with high oxidative stress and inflammation, that directly affects nitric oxide (NO) bioavailability and promotes vascular dysfunction and plaque formation (36). Several factors such as smoking, obesity, age, hypertension, hyperlipidemia, physical inactivity, and poor dietary habits, have been identified to adversely affect the endothelium (37). It seems that impaired endothelial function is the initial step in atherogenesis, which is consider an important factor in the development of CVD 
(38-40). Clinical manifestations of endothelial dysfunction include edema, hypertension, abnormal vasoconstriction, and hypercoagulability.

NO is synthesized from L-arginine by NO synthase (NOS). Cardiac myocytes express two types of NOS, endothelial NOS (eNOS) and inducible NOS (iNOS). Shear stress stimulate the production of NO via the eNOS, while inflammatory cytokines does do so via the proinflammatory iNOS. Disruption of NO contributes to systemic vasoconstriction and increases vascular stiffness which are hallmarks in HF. As a result, there is an increase in left and right ventricular systolic workload. In addition, the basal production of NO due to stimulation of iNOS is increased in patients with HF. However, high concentrations of NO have been associated with myocyte injury and reduced myocardial contractility. Contrarily, the reduced $\mathrm{CO}$ associated with HF, limits endothelial shear stress which is responsible for the stimulation of eNOS expression. Downregulation of eNOS results in reduced NO bioavailability and consequently lower levels of vasodilatory response $(5,6)$.

Several invasive and non-invasive techniques have been developed to evaluate endothelial function. However, the gold standard to assess its function and its impairment continues to be unclear (39). Flow-mediated dilation (FMD) is a common non-invasive tool for the assessment of endothelial function/dysfunction that has shown to be accurate and reproducible, allowing for repetition of measurements over time (39-41). It indirectly measures NO release in response to shear stress due to reactive hyperemia. FMD relies on obtaining a baseline diameter of the artery with ultrasound imaging before cuff inflation to supra-systolic pressure. Subsequent cuff deflation induces reactive hyperemia with shear stress causing vasodilatation. The change in artery diameter is then quantified as a percentage from the baseline diameter. However, FMD requires specialized 
and expensive equipment, as well as highly trained operators, and the technique is not standardized $(6,42)$.

Agewall et al. (43) compared FMD in the brachial and radial arteries after reactive hyperemia induced by forearm or upper-arm cuff occlusion in 24 healthy subjects. They found that FMD was greater in the radial artery than in the brachial artery. It seems that FMD is greater in smaller arteries maybe due to greater hyperemic shear stress in response to the same stimulus. Also, they found that FMD of the brachial artery was significantly greater after upper-arm occlusion compared to forearm occlusion, despite similar increases in blood flow. This may be explained by the influence of other components not mediated by NO, most probably related to local tissue ischemia (e.g., endothelial-derived hyperpolarizing factor, potassium, adenosine).

Similarly, Doshi et al. (42) compared cuff positions (wrist or upper arm occlusion) to evaluate the NO component of brachial artery dilatation in 10 healthy males. They found that dilatation was significantly greater after upper-arm occlusion and was only partially attenuated after infusion of a NOS inhibitor. Contrarily, dilatation after wrist occlusion was abolished after the infusion of the NOS inhibitor. They concluded that FMD of the brachial artery following forearm occlusion was mediated exclusively by NO. For this reason, FMD following wrist occlusion may be a more valid marker of endothelial function than dilatation following upper-arm occlusion.

Several studies have shown that FMD is an independent predictor of future CVD events, including HF. In addition, in HF patients a reduction in FMD has been shown to be an important predictor of adverse outcomes associated with higher rates of hospitalization, and mortality (4446). The study of Ärnlöv et al. (35) revealed that individuals in the highest quartile of FMD had a 47\% lower HF risk compared with individuals in the lowest quartile. In addition, they reported 
lower risk according to higher FMD for HFrEF, but not for HFpEF (HR 0.79, 95\% CI 0.64-0.97 vs. $0.99,95 \%$ CI $0.78-1.26$, respectively).

In a meta-analysis conducted by Ras et al. (47) they found an inverse association between FMD and future CVD events, with a stronger relation in diseased populations. The overall CVD risk was 0.92 (95\%CI: 0.88; 0.95) per 1\% higher FMD. Comparably, the meta-analysis of Inaba et al. (48) reported a 15\% lower risk of CVD events per $1 \%$ increase in brachial FMD.

A study conducted by Kishimoto et al. (34) revealed that individuals with HFpEF had significantly smaller FMD values when compared with their healthy counterparts $(2.9 \pm 2.1 \%$ and $4.6 \pm 2.7 \% \mathrm{p}<0.001$, respectively). Even after adjustment for age, sex, hypertension, dyslipidemia, and diabetes, the relationship between FMD and HFpEF remained significant. In agreement, a study among HFpEF patients showed lower brachial artery FMD values when compared to control subjects (median 3.6\% versus 7.2\%, respectively). The relationship between brachial artery FMD and HFpEF remained significant after adjustment for beta-adrenergic blockade, treatment with loop diuretics therapy, and history of CAD (49).

Recent evidence suggests that reduced endothelium-dependent vasodilation in HF may be a reflection of the underlying risk factors, rather than ventricular function in itself. Hashimoto et al. (38) found that endothelial function was impaired according to the accumulation of risk factors. FMD was significantly lower in those with one or more coronary risk factors when compared to control subjects.

Endothelial dysfunction often accompanies diabetes and hypertension, both important risk factors for HFpEF (23). These conditions cause oxidative stress with high levels of reactive oxygen species that interfere with NO production in endothelial cells (50). Disruption of the NO signaling 
pathway can therefore explain the development of concentric LV remodeling, increased stiffness of the cardiomyocyte, and increased collagen deposition in HFpEF subjects (23).

Atrial fibrillation which is another risk factor for HF especially in those with HFpEF, has been associated with endothelial dysfunction. A study of 2,936 participants from MESA, revealed an inverse relationship between FMD and AF. They reported that for 1-standard deviation (SD) increase in FMD (2.8\%) there was a $16 \%$ lower risk of $\mathrm{AF}(\mathrm{HR}=0.84,95 \%$ CI $0.70-0.99)$, suggesting a role for endothelial dysfunction in AF pathogenesis (51).

An alternate option to the traditional FMD is total brachial artery reactivity (TBAR), a noninvasive measurement that evaluates endothelial function using ultrasound imaging. This measurement uses a baseline brachial artery diameter following cuff deflation instead of before cuff inflation. It compensates for the absence of a stereotactic device, limiting the errors associated with ultrasound probe displacement (52). Although the measurement process is different from FMD, they might be comparable and equivalent. Ostrem et al. (40) compared pre-occlusion, occlusion, and post-occlusion baseline brachial artery measurements on the calculation of FMD in 418 children and 533 adults. They found non-significant differences between the three baseline measurements of brachial artery diameter. Additionally, peak FMD values were not significantly different when calculated from pre-occlusion, occlusion or post-occlusion baseline diameters in both, children and adults.

Polak et al. (52) evaluated whether TBAR was associated with first time coronary heart disease (CHD) events among participants of MESA. Increased TBAR was associated with lower risk of CHD events with a $\mathrm{HR}$ of 0.78 per SD increase $(9.7 \mathrm{SD} ; \mathrm{p}=0.001)$. Those participants with TBAR above the median of $7.87 \%$ had a $31 \%$ lower risk of CHD events (HR 0.69; 95\% C.I. 0.550.87; $\mathrm{p}=0.001$ ). They found TBAR to be an independent predictor of incident $\mathrm{CHD}$ events. 


\section{SUMMARY}

In summary, evidence suggests that endothelial dysfunction may contribute to the pathogenesis and maintenance of $\mathrm{HF}$ and may be a potential target for new therapeutic development. Nonetheless, the questions about whether endothelial dysfunction, assessed by TBAR, is of difference importance among HF subtypes, and whether it is a cause or a consequence remain unanswered. Determining the relationship between endothelial dysfunction measured by TBAR and incident HF subtypes may help to elucidate a better understanding of the complex syndrome that is HF. This may contribute to the establishment of guidelines that recommend clinically testing endothelial function in addition to traditional risk factors. 


\section{REFERENCES}

1. Yancy C, Jessup M, Bozkurt B, et al. 2013 ACCF/AHA guideline for the management of heart failure: Executive summary: A report of the American college of cardiology foundation/American heart association task force on practice guidelines. Circulation. 2013;128(16):1810-1852.

2. Kemp CD, Conte JV. The pathophysiology of heart failure. Cardiovasc Pathol. 2012; 21:365-371.

3. Alem MM. Endothelial dysfunction in chronic heart failure: assessment, findings, significance, and potential therapeutic targets. Int. J. Mol. Sci. 2019; 20(13):3198.

4. Jackson G, Gibbs CR, Davies MK, Lip GYH. ABC of heart failure. Pathophysiology. BMJ. 2000; 320:167-170.

5. Bauersachs J, Widder JD. Endothelial dysfunction in heart failure. Pharmacol Rep. 2008. 60(1):119-126.

6. Giannitsi S, Mpougiaklh M, Bechlioulis A, Naka K. Endothelial dysfunction and heart failure: A review of the existing bibliography with emphasis on flow mediated dilation. JRSM Cardiovasc Dis. 2019; 8:1-7.

7. Ziaeian B, Fonarow GC. Epidemiology and aetiology of heart failure. Nat Rev Cardiol. 2016; 13(6):368-378.

8. Tomasoni D, Adamo M, Lombardi CM, Metra M. Highlights in heart failure. ESC Heart Fail. 2019;6:1105-1127.

9. Ponikowski P, Voors AA, Anker SD, et al. 2016 ESC guidelines for the diagnosis and treatment of acute and chronic heart failure: the task force for the diagnosis and treatment of acute and chronic heart failure of the European Society of Cardiology 
(ESC) developed with the special contribution of the Heart Failure Association (HFA) of the ESC. 2016; 37(27): 2129-2200.

10. Lee MP, Glynn RJ, Schneeweiss S, et al. Risk factors for heart failure with preserved or reduced ejection fraction among Medicare beneficiaries: Applications of competing risks analysis and gradient boosted model. Clin Epidemiol. 2019; 12:607-616.

11. Kurmani S, Squire I. Acute heart failure: definition, classification and epidemiology. Curr Heart Fail Rep. 2017;14(5):385-392.

12. Roger VL. Epidemiology of heart failure. Circ Res. 2013; 113(6): 646-659.

13. Mosterd A, Hoes AW. Clinical epidemiology of heart failure. Heart.2007; 93(9): 11371146.

14. Pfeffer MA, Shah AM, Borlaug BA. Heart failure with preserved ejection fraction in perspective. Circ Res. 2019; 124(11):1598-1617.

15. Bui AL, Horwich TB, Fonarow GC. Epidemiology and risk profile of heart failure. Nat Rev Cardiol. 2011; 8(1):30-41.

16. McKee PA, Castelli WP, McNamara PM, Kannel WB. The natural history of congestive heart failure: The Framingham study. N Engl J Med. 1971; 285(26):14411446.

17. Carlson KJ, Lee DC, Goroll AH, et al. An analysis of physicians' reasons for prescribing long-term digitalis therapy in outpatients. J Chronic Dis. 1985; 38(9):733739.

18. Eriksson H, Caidahl K, Larsson B, et al. Cardiac and pulmonary causes of dyspneavalidation of a scoring test for clinical-epidemiological use: The study of men born in 1913. Eur Heart J. 1987; 8(9):1007-1014. 
19. Swedberg K, Cleland J, Dargie H, et al. Guidelines for the diagnosis and treatment of chronic heart failure: Executive summary (update 2005): The task force for the diagnosis and treatment of chronic heart failure of the European society of cardiology. Eur Heart J. 2005; 26:1115-1140.

20. Borlaug BA, Redfield MM. Diastolic and systolic heart failure are distinct phenotypes within the heart failure spectrum. Circulation. 2011; 123(18):2006-13.

21. Gevaert AB, Boen JRA, Segers VF, Van Craenenbroeck EM. Heart failure with preserved ejection fraction: a review of cardiac and noncardiac pathophysiology. Front Physiol. 2019;10:638.

22. Parikh KS, Sharma K, Fiuzat M, et al. Heart failure with preserved ejection fraction expert panel report. JACC Heart fail. 2018; 6(8):619-632.

23. Komajda M, Lam CSP. Heart failure with preserved ejection fraction: a clinical dilemma. Eur Heart J. 2014; 35:1022-1032.

24. Chahal H, Bluemke DA, Wu CO, et al. Heart failure risk prediction in the Multi-Ethnic Study of Atherosclerosis. Heart. 2015;101(1):58-64.

25. He J, Ogden LG, Bazzano LA, et al. Risk Factors for congestive heart failure in US men and women: NHANES I epidemiologic follow-up study. Arch intern Med. 2001; 161(7):996-1002.

26. Dunlay SM, Weston SA, Jacobsen SJ, et al. Risk factors for heart failure: a populationbased case-control study. Am J Med. 2009; 122(11):1023-1028.

27. Ho JE, Enserro D, Brouwers FP, et al. Predicting heart failure with preserved and reduced ejection fraction: The International Collaboration on heart failure subtypes. Circ Heart Fail. 2016; 9(6):1-20. 
28. King M, Kingery J, Casey B. Diagnosis and evaluation of heart failure. Am Fam Physician. 2012; 85(12):1161-1168.

29. Wang TJ, Larson MG, Levy D, et al. Plasma natriuretic peptide levels and the risk of cardiovascular events and death. N Engl J Med. 2004; 350:655-663.

30. Mahmood SS, Wang TJ. The epidemiology of congestive heart failure: the Framingham Heart Study perspective. Glob Heart. 2013; 8(1):77-82.

31. Lloyd-Jones DM, Larson MG, Leip EP, et al. Lifetime risk for developing congestive heart failure: the Framingham Heart Study. Circulation. 2002; 106(24):3068-72.

32. Ho JE, Lyass A, Lee DS, et al. Predictors of new-onset heart failure: differences in preserved versus reduced ejection fraction. Circ Heart Fail. 2012; 6(2):279-286.

33. Lee DS, Gona P, Vasan RS, et al. Relation of disease pathogenesis and risk factors to heart failure with preserved or reduced ejection fraction: insights from the Framingham heart study of the national heart, lung, and blood institute. Circulation. 2009; 119(24):3070-7.

34. Kishimoto S, Kajikawa M, Maruhashi T, et al. Endothelial dysfunction and abnormal vascular structure are simultaneously present in patients with heart failure with preserved ejection fraction. International Journal of Cardiology. 2016; 231:181-7.

35. Ärnlöv J, Sang Y, Ballew SH, et al. Endothelial dysfunction and the risk of heart failure in a community-based study: the Multi-Ethnic Study of Atherosclerosis. ESC Heart Fail. 2020; 7(6):4231-4240.

36. Irace C, Tripolino C, Scavelli FB, et al. Brachial low-flow-mediated constriction is associated with delayed brachial flow-mediated dilation. J Atheroscler Thromb. 2015; 22:000-000. 
37. Widmer R.J, Lerman A. Endothelial dysfunction and cardiovascular disease. Glob Cardiol Sci Pract. 2014; 2014(3):291-308.

38. Hashimoto M, Kozaki K, Eto M, et al. Association of coronary risk factors and endothelium-dependent flow-mediated dilatation of the brachial artery. Hypertens Res. $2000 ; 23(3): 233-238$.

39. Chong AY, Blann AD, Lip GYH. Assessment of endothelial damage and dysfunction: observations in relation to heart failure. $Q J$ Med. 2003; 96:253-267.

40. Ostrem JD, Dengel DR, Marlatt KL, et al. Comparison of baseline brachial artery measurements and effect on peak flow-mediated dilation. Clin Physiol Funct Imaging. $2015 ; 35(1): 34-40$.

41. Green DJ, Jones H, Thijssen D, et al. Flow-mediated dilation and cardiovascular event prediction. Does nitric oxide matter? Hypertension. 2011; 57(3):363-369.

42. Doshi SN, Naka KK, Payne N, et al. Flow-mediated dilatation following wrist and upper arm occlusion in humans: the contribution of nitric oxide. Clin Sci. 2001;101(6):629-35.

43. Agewall S, Doughty RN, Bagg W, et al. Comparison of ultrasound assessment of flowmediated dilatation in the radial and brachial artery with upper and forearm cuff positions. Clin Physiol. 2000; 21(1): 9-14.

44. Areas GPT, Mazzuco A, Caruso FR, et al. Flow-mediated dilation and heart failure: a review with implications to physical rehabilitation. Heart Fail Rev. 2019; 24(1):69-80.

45. Shechter M, Issachar A, Marai I, et al. Long-term association of brachial artery flowmediated vasodilation and cardiovascular events in middle-aged subjects with no apparent heart disease. Int J Cardiol. 2009; 134(1):52-58. 
46. Yeboah J, Folsom AR, Burke GL, et al. Predictive value of brachial flow-mediated dilation for incident cardiovascular events in a population-based study: The multiethnic study of atherosclerosis. Circulation. 2009; 120(6):502-509.

47. Ras RT, Streppel MT, Draijer R, et al. Flow-mediated dilation and cardiovascular risk prediction: A systematic review with meta-analysis. Int J Cardiol. 2012; 168:344-351.

48. Inaba Y, Chen JA, Bergmann SR. Prediction of future cardiovascular outcomes by flow-mediated vasodilatation of brachial artery: A meta-analysis. Int J Cardiovasc Imaging. 2010; 26(6):631-640.

49. Maréchaux S, Samson R, Van Belle E, et al. Vascular and microvascular endothelial function in heart failure with preserved ejection fraction. J Card Fail. 2016; 22(1):311.

50. Hadi HAR, Suwaidi JAI. Endothelial dysfunction in diabetes mellitus. Vasc Health Risk Manag. 2007; 3(6):853-76.

51. O’Neal WT, Efird JT, Yeboah J, Nazarian S, et al. Brachial flow-mediated dilation and incident atrial fibrillation: the multi-ethnic study of atherosclerosis. Arterioscler Thromb Vasc Biol. 2014; 34(12): 2717-2720.

52. Polak J, Ouyang P, Vaidya D. Total brachial artery reactivity and first time incident coronary heart disease events in a longitudinal cohort study: The multi-ethnic study of atherosclerosis. PLoS ONE. 2019; 14(4): e0211726. 
Chapter Three: Methodology 
The purpose of this study was to investigate whether total brachial artery reactivity (TBAR) is associated with overall incident heart failure (HF) and the two HF sub-types, heart failure with reduced ejection fraction (HFrEF) and heart failure with preserved ejection fraction (HFpEF), in a diverse population-based sample of U.S. adults. This chapter provides the details of the methodology used to address the research question.

\section{DATA COLLECTION}

The Multi-Ethnic Study of Atherosclerosis (MESA) is an ongoing, multicenter, prospective cohort study of cardiovascular disease (CVD) sponsored by the National Heart Lung and Blood Institute of the National Institutes of Health. Specific information on the MESA protocol has been described elsewhere (1), and additional information can be found at https://www.mesa-nhlbi.org/. The MESA study a diverse, population-based sample of 6,814 men and women (45-84 years of age), with no clinical CVD history at baseline. The cohort includes Caucasian (38\%), African American (28\%), Hispanic (22\%), and Asian (12\%) participants recruited from six field centers across the United States between July 2000 and August 2002:

1. University of California, Los Angeles, CA

2. University of Minnesota, St. Paul, MN

3. Northwestern University, Chicago, IL

4. Wake Forest University, Winston-Salem, NC

5. John Hopkins University, Baltimore, MD

6. Columbia University, New York, NY 
Eligibility status was determined from self-reported information. MESA's exclusion criteria included:

1. Age younger than 45 or older than 84 years

2. Physician-diagnosed:
a. Heart attack
b. Angina or taking nitroglycerin
c. Stroke or transient ischemic attack
d. Heart failure

3. Current atrial fibrillation

4. Having undergone procedures related to CVD

5. Active treatment for cancer

6. Pregnancy

7. Any serious medical condition which would prevent long-term participation

8. Weight $>300$ pounds

9. Cognitive inability as judged by the interviewer

10. Living in a nursing home or on the waiting list for a nursing home

11. Plans to leave the community within five years

12. Language barrier (speaks other than English, Spanish, Cantonese or Mandarin)

13. Chest CT scan in the past year

Six additional exams have been completed since 2000, and participants are contacted every nine to 12 months to assess clinical morbidity and mortality. For the present study, the sample included 5,499 participants (45-84 years of age) with available brachial artery endothelial function assessed by ultrasound at the baseline visit. 
The present study was reviewed and approved by the Institutional Review Board of the University of North Florida (Appendix A). Data from the MESA was requested and obtained from the National Institutes of Health/ National Heart, Lung, and Blood Institute: Biologic Specimen and Data Repository Information Coordinating Center (2).

\section{PRIMARY DEPENDENT VARIABLE}

The primary outcome was time to congestive heart failure (TTCHF) defined as the number of days from enrollment to congestive heart failure (CHF) event. CHF was an adjudicated event classified as 'Definite', 'Probable', or 'Absent'. MESA identified possible HF events in both inpatient and outpatient mainly by participant self-report via follow-up calls, notification at clinic visits, or directly to the field center. Other means of identifying HF events included a search in the National Death Index (NDI), obituaries, or other public notice. At least two physicians reviewed medical records and death certificates, interviews, questionnaires, and other procedures of the eligible events to assign a final classification. Probable HF required the presence of HF symptoms, a physician diagnosis of HF, and evidence of the patient receiving medical treatment for HF such as diuretics, digitalis, vasodilators, beta-blockers, or ACE inhibitors. Definite HF required the same criteria as probable $\mathrm{HF}$ and one or more of the following criteria:

1. Pulmonary edema/congestion by chest x-ray.

2. Dilated ventricle or poor left ventricular function by echocardiography or ventriculography.

3. Evidence of left ventricular diastolic dysfunction. 
Those who did not meet any criteria or only had a physician diagnosis of HF but without any treatment or evidence on imaging were classified as absent. Disagreement or conflicting endpoint diagnosis were assigned to a third reviewer, or if necessary, to the MESA morbidity and mortality committee for the final decision. For those participants with a final classification of HF, ejection fraction (EF) was recorded either as a percent or classified as 'Normal', 'Low', or 'Unknown'. For this study, incident HF overall included participants with either definite or probable HF, independent of EF. Participants with $\mathrm{EF} \leq 40 \%$ or classified as 'Low' were considered HFrEF, and those with $\mathrm{EF} \geq 50 \%$ or classified as 'Normal' were considered HFpEF.

\section{PRIMARY INDEPENDENT VARIABLE}

The primary independent variable in this study was TBAR1, defined as the total brachial artery reactivity as a percent (\%) value attained by ultrasound imaging. Participant preparation included abstaining from food and caffeine for six hours prior to the brachial artery endothelial function assessment. If necessary, a small snack, mostly carbohydrates and no fat content, was provided 90 minutes prior to the assessment. Consumption of vitamin $\mathrm{E}$ or $\mathrm{C}$ in the six hours before the procedure was discouraged, and the participant should not have smoked any cigarettes (3). Blood pressure was then obtained in both the right and left arms using the automated sphygmomanometer (Dinamap ${ }^{\circledR}$, Tampa, FL).

Trained technicians at each of the six field centers acquired B-mode ultrasound images with a Logiq-700 ultrasound device (GE Healthcare, Waukesha, WI) and an ultrasound transducer (M12L) set at $9 \mathrm{MHz}$, without the use of a stereotactic holder. Participants were in a supine position during the examination with the right arm supported at the elbow and wrist. The ultrasound probe 
was placed on the right arm's medial aspect a few centimeters above the elbow with a slight angulation to best visualize the brachial artery. An occlusion cuff was placed on the upper right forearm just below the antecubital fossa and inflated to a pressure of $200 \mathrm{mmHg}$ when the baseline SBP was $<150 \mathrm{mmHg}$, or $50 \mathrm{mmHg}$ above maximal systolic pressure when the baseline SBP was $>150 \mathrm{mmHg}$ but, $<180 \mathrm{mmHg}$. The cuff was kept inflated for five minutes with the ultrasound probe held centered over the same brachial artery segment. Images were videotaped and recorded using super VHS tapes, starting 15 seconds before cuff deflation, and continuing for 90 seconds after cuff release.

Images were sent to Tufts Medical Center Ultrasound Reading Center for blinded processing. Digital streams of the brachial artery ultrasound images were acquired from the videotapes at a frame-rate of 30 frames-per-second as MJPEG compressed images (compression ratio six to one) using a Pinnacle DC-30 Video board (Corel Inc., Mountain View CA) and a Compaq AP-200 workstation (Compaq Computer Corporation, Houston, TX) equipped with a Pentium III processor (Intel Corporation, Santa Clara, CA). A reader reviewed the images and identified the point at which the blood pressure cuff had been released. The reader then identified an appropriate brachial artery segment and placed a rectangular region of interest on a selected image frame (Figure 1). 


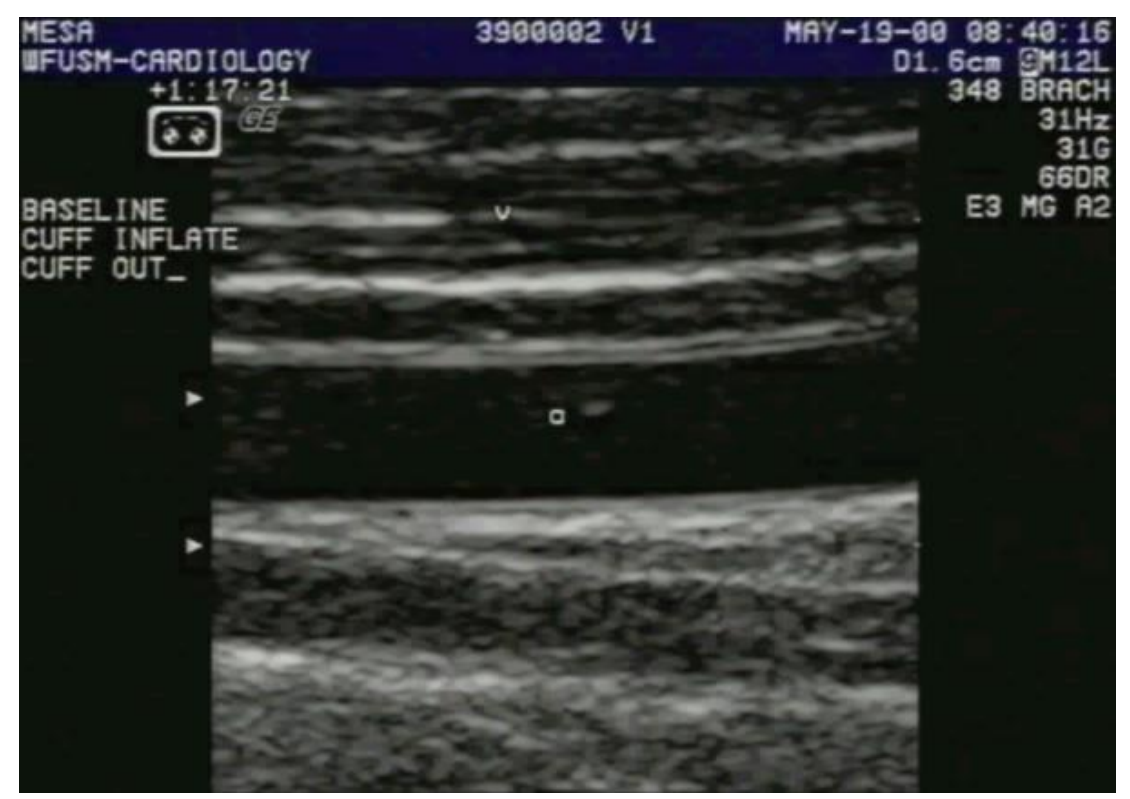

Fig. 1 Segment of the brachial artery. From "MESA manual of operations: field center and laboratory procedures” by O’Leary D. H. [cited 2021 January].

Customized software was used to calculate the location of the near and far wall mediaadventitia interfaces in this region of interest and to generate brachial artery diameter versus time curves without manual editing. These were transferred to Access (Microsoft, Redmond WA) databases for archiving. The archived brachial artery diameter curves were subsequently retrieved and processed using a MATLAB (The MathWorks Inc., Natick MA) program that smoothed the diameter versus time curves using a finite impulse response digital filter and processed the resultant curves to identify the maximum diameter and time to maximum diameter starting 20 seconds following the release of the blood pressure cuff. The algorithm then searched for a minimum diameter going backwards until 10 seconds after cuff release based on previous observations. 
Then TBAR was calculated as:

$\operatorname{TBAR}(\%)=\underline{(\text { maximum diameter }- \text { minimum diameter })} \times 100$

(minimum diameter)

Although 5,499 brachial diameter curves were processed, there were 765 instances where the curve analysis algorithm detected a maximum diameter in a time window 20 to 30 seconds after cuff release and failed to find a smaller diameter in the preceding time interval. These cases likely represented low amplitude responses and were assigned a $0 \%$ TBAR value (4).

A dichotomous TBAR variable was created based on the median value (below or above 7.9\%). Analysis in this study was performed after excluding potential TBAR outliers. We set two limits, a minimum value at $0 \%$ and a maximum value at the $99^{\text {th }}$ percentile $(40.9 \%)$.

\section{OTHER INDEPENDENT VARIABLES}

The potential confounding variables that were controlled for in this study include the following:

$A G E$

Age was self-reported on the personal history form at exam one. This was included in the analysis as a continuous variable.

\section{SEX}

Sex was self-reported on the personal history form at exam one as either male or female. 


\section{RACE/ETHNICITY}

Race was self-reported as either Caucasian, African American, Hispanic, or Asian.

\section{SMOKING}

Smoking status was self-reported on the personal history form at exam one and was a created variable. Participants were classified as never smokers, former smokers, or current smokers. Those who answered "yes" to the question, "Have you smoked cigarettes in the past 30 days?" were considered current smokers. Those who answered "no" to having smoked cigarettes in the past 30 days but "yes" to the question, "Have you smoked at least 100 cigarettes in your lifetime?" were considered former smokers. Those who answered "no" to having smoked at least 100 cigarettes in their lifetime and "no" to having smoked cigarettes in the past 30 days were considered never smokers.

\section{BODY MASS INDEX}

Body mass index (BMI) was calculated as body weight in kilograms $(\mathrm{kg})$ divided by the height in meter squared $\left(\mathrm{m}^{2}\right)$ using measurements obtained at exam one. The four BMI categories used in this study included normal $\left(<25 \mathrm{~kg} / \mathrm{m}^{2}\right)$, overweight $\left(\geq 25-<30 \mathrm{~kg} / \mathrm{m}^{2}\right)$, obese $(\geq 30-\leq 40$ $\left.\mathrm{kg} / \mathrm{m}^{2}\right)$, and extreme obese $\left(>40 \mathrm{~kg} / \mathrm{m}^{2}\right)$.

\section{BLOOD PRESSURE}

Blood pressure was measured in the seated position using a Dinamap Monitor Pro 100® (Critikon, Tampa, Florida, USA) automated oscillometric device; pressures were the average of the last two of three performed measurements. Blood pressure was categorized into three groups according to the most recent blood pressure recommendations by the American Heart Association 
(5): normal (SBP $<120 \mathrm{mmHg}$ and $\mathrm{DBP}<80 \mathrm{mmHg}$ ), elevated (SBP 120-129 $\mathrm{mmHg}$ and DBP $<80 \mathrm{mmHg}$ ), and hypertensive (SBP $\geq 130 \mathrm{mmHg}$ or $\mathrm{DBP} \geq 80 \mathrm{mmHg}$ ). Participants who reported using antihypertensive medications were also considered as being hypertensive.

\section{LOW-DENSITY LIPOPROTEIN CHOLESTEROL}

The low-density lipoprotein cholesterol (LDL-C) was calculated using the formula of Friedewald et al. (6). In MESA, it was a created variable and was categorized according to the National Cholesterol Education Program Guidelines (NCEP) (7). LDL-C values $\geq 130 \mathrm{mg} / \mathrm{dL}$ were classified as elevated. Additionally, lipid-lowering medication use was self-reported on the medications form at exam one and confirmed during the medication interview. Participants taking lipid-lowering medications were also classified as having elevated LDL-C.

\section{HIGH-DENSITY LIPOPROTEIN CHOLESTEROL}

The high-density lipoprotein cholesterol (HDL-C) was measured in EDTA plasma using the cholesterol oxidase method (Roche Diagnostics) after precipitation of non-HDL-cholesterol with magnesium/dextran. HDL-C was used to create a gender-stratified variable according to the NCEP Guidelines (7). HDL-C values $<50 \mathrm{mg} / \mathrm{dL}$ for females and $<40 \mathrm{mg} / \mathrm{dL}$ for males were classified as low. Participants taking lipid-lowering medications were also classified as having low HDL-C.

\section{TRIGLYCERIDES}

Triglycerides were measured in EDTA plasma using Triglyceride GB reagent (Roche Diagnostics, Indianapolis, IN 46250) on the Roche COBAS FARA centrifugal analyzer. In the MESA, triglycerides were categorized according to the NCEP Guidelines (NCEP) (7). 
Triglyceride values $\geq 150 \mathrm{mg} / \mathrm{dL}$ were classified as elevated. Participants taking lipid-lowering medications were also classified as having elevated triglycerides.

\section{DIABETES}

The presence of diabetes mellitus was based on self-reported physician diagnosis, use of insulin or oral hypoglycemic medication, or a fasting glucose value $\geq 126 \mathrm{mg} / \mathrm{dL}$ (8). Those who answered "yes" to the question, "Has a doctor ever told you that you had diabetes (sugar in the blood)?" were considered as having diabetes. Those who answered "yes" to the question, "Are you taking medicine (insulin or pills) for this?" were also considered as having diabetes. Serum glucose was measured by rate reflectance spectrophotometry using thin-film adaptation of the glucose oxidase method on the Vitros analyzer (Johnson \& Johnson Clinical Diagnostics, Inc., Rochester, NY 14650).

\section{MYOCARDIAL INFARCTION}

Identification of possible MI events in both inpatient and outpatient were made by participant self-report via follow-up calls, notification at clinic visits, or directly to the field center. Other means of identifying MI events included search in the NDI, obituaries, or other public notice. At least two physicians reviewed each event's case materials, and its data were analyzed by a computer algorithm that used standardized criteria to assign a final classification. The criteria for MI included information about chest pain, cardiac enzymes, and electrocardiogram. For the analysis, we included only MI events that occurred before the first HF event. 


\section{ATRIAL FIBRILLATION}

AF was either self-reported or through review of in-hospital events records via the International Classification of Diseases (ICD9) code (427.31: AF). For the analysis, we included only AF events that occurred before the first HF event.

\section{STATISTICAL ANALYSIS}

The data in this study were managed using SAS 9.4 (SAS Institute Inc, Cary, NC) (9). Variable recodes, data coding validation and analysis were conducted in SAS. Variables are presented as means and standard deviation (SD) values if continuous and as percentages if categorical. Descriptive characteristics were obtained using procedures PROC MEANS and PROC FREQ for continuous and categorical variables, respectively. The procedure PROC UNIVARIATE was used to calculate TBAR outliers. We set two limits, a minimum value at $0 \%$ and a maximum value at the $99^{\text {th }}$ percentile. A dichotomous TBAR variable was created based on the median value (below or above $7.9 \%$ ).

Separate proportional hazards regression models (PROC PHREG) were used to calculate multivariable-adjusted hazard ratios (HR) and 95\% confidence intervals $(\mathrm{CI})$ to determine the risk of HF overall, HFrEF, and HFpEF according to TBAR values. HF participants without EF data were excluded from the subtype analyses. Three different models were created controlling for known HF risk factors, including age, gender, race/ethnicity, BMI, diabetes, blood pressure, HDLC, LDL-C, triglycerides, smoking status, MI, and AF. Model 1 was unadjusted, model 2 was ageadjusted, and model 3 was fully adjusted. The level of significance was set at $p=0.05$ for all tests. 
Additionally, using proportional hazards regression models, covariates included in the fully adjusted model were chosen using a stepwise backward elimination process; covariates that did not contribute significantly based on $\mathrm{p}=0.05$ were removed and excluded from the final analysis. A final parsimonious model was included to elucidate the relationship between the independent and dependent variables. Kaplan-Meier survival curves were plotted by the median value of TBAR for overall HF, HFrEF, and HFpEF, using the life test procedure (PROC LIFETEST), and compared statistically using the log-rank test.

Finally, we evaluated the effect of TBAR as a continuous variable (per 1 SD value of 9.7\%) on time to $\mathrm{HF}$, using a cut point of $50 \%$ for EF. The analysis in this study included a whole sample of 5,499 participants with available TBAR values. We also performed the analysis excluding the 765 curves with low amplitude responses for a full sample of 4,734 participants. 


\section{REFERENCES}

1. Bild DE, Bluemke DA, Burke GL, et al. Multi-Ethnic Study of Atherosclerosis: objectives and design. Am J Epidemiol. 2002; 156(9):871-881.

2. National Institutes of Health/National Health Lung and Blood Institute: Biologic Specimen and Data Repository Information Coordinating Center. In: BIOLINCC. https://biolincc.nhlbi.nih.gov/home/.

3. MESA Coordinating Center. MESA manual of operations: field center and laboratory $\left.\begin{array}{lllll}\text { procedures. } & \text { [cited } & 2021 & \text { January }\end{array}\right]$ Available from http://www.mesanhlbi.org/publicDocs/MesaMop/MesaMop1-5-01.doc.

4. Polak J, Ouyang P, Vaidya D. Total brachial artery reactivity and first time incident coronary heart disease events in a longitudinal cohort study: The multi-ethnic study of atherosclerosis. PLoS ONE. 2019; 14(4): e0211726.

5. Whelton PK, Carey RM, et al. 2017 Guideline for the prevention, detection, evaluation, and management of high blood pressure in adults. JACC. 2018; 71(19): e127-248.

6. Friedewald WT, Levy RI, Fredrickson DS. Estimation of the concentration of low-density lipoprotein cholesterol in plasma, without use of the preparative ultracentrifuge. Clin Chem. 1972; 18: 499-502.

7. National Cholesterol Education Program (NCEP) Expert Panel on detection, evaluation, and treatment of high blood cholesterol in adults (adult treatment panel III). JAMA. 2001; 285(19): 2486-97.

8. Genuth S, Alberti KGMM, Bennett P, et al. Follow-up report on the diagnosis of diabetes mellitus. Diabetes Care. 2003; 26(11): 3160-7. 
9. SAS Institute Inc. 2016. Base SAS ${ }^{\circledR} 9.4$ Procedures Guide: Statistical Procedures, Sixth Edition. Cary, NC: SAS Institute Inc. 
Chapter Four: Manuscript 
TOTAL BRACHIAL ARTERY REACTIVITY AND INCIDENT HEART FAILURE AND HEART FAILURE SUB-TYPES: MULTI-ETHNIC STUDY OF ATHEROSCLEROSIS 
Background:

Methods:

\section{Results:}

Over a mean follow-up period of 12.5 years, incident HF was diagnosed in 250 participants; 98 classified as HFrEF, 106 as HFpEF, and 46 with 
unknown or borderline EF (41-49\%). Crude analysis revealed that those with TBAR below the median had a significantly greater risk of HF (HR 1.46; 95\% CI 1.13-1.88, $\mathrm{p}<0.01$ ) and HFrEF (HR 1.61; 95\% CI 1.07-2.43, $\mathrm{p}<0.05$ ). Following adjustment for known HF risk factors (e.g., age, sex, race, blood pressure), these relationships were no longer statistically significant. Borderline significant results were revealed in those with HFpEF (HR 1.43; 95\% CI 0.97-2.12, p=0.06). Kaplan-Meier curves suggest significantly lower risks of developing HF and HFrEF in those with TBAR above the median (log-rank $\mathrm{p} \leq 0.05$ for both). When examined as a continuous variable, with a cut point of $50 \%$ for EF, every 1-standard deviation (9.7\%) increase in TBAR resulted in a 19\% and 29\% decrease in risk of $\mathrm{HF}(\mathrm{p}<0.05)$ and $\operatorname{HFrEF}(\mathrm{p}=0.05)$, respectively.

Conclusion: $\quad$ Lower TBAR values were associated with higher rates of incident $\mathrm{HF}$ and HFrEF, suggesting a possible role of endothelial dysfunction in HF pathogenesis. The impact of other known HF risk factors may mediate this relationship, thus further research is warranted. 


\section{INTRODUCTION}

Heart failure (HF) is a complex clinical syndrome that is associated with markedly altered hemodynamic, neurohumoral, and peripheral vascular responses $(1,2)$. The structural and functional disturbances associated with HF affect the ability of the ventricles to deliver oxygenated blood to tissues, resulting in significant morbidity, mortality, and healthcare expenses, especially among those aged 65 and older (2,3). Based on systolic function measured by left ventricular ejection fraction (LVEF), HF can be classified into two major subtypes: HF with reduced EF (HFrEF), and HF with preserved EF (HFpEF). Despite the efforts to establish clear differences between them, the etiology, pathophysiology, and effective treatments for HFpEF are still unclear (12).

Heart failure affects $1-3 \%$ of the population worldwide and rises up to $10 \%$ and more in populations over 70 years old (4). A recent report from the American Heart Association estimates that 6.2 million adults in the United States have heart failure, and approximately half of those events are characterized by reduced ejection fraction and the other half by preserved ejection fraction (5). Although efforts to find effective treatment have reduced the prevalence of rehospitalizations to $30-50 \%$, the mortality rates remain high (4). In the Atherosclerosis Risk in Communities study, the 30-day, 1-year, and 5-year case fatality rates after hospitalization for HF were $10 \%, 22 \%$, and $42 \%$, respectively (29).

Endothelial dysfunction has been proposed as a phenotypic expression of HF as it may play a role in its pathogenesis $(5,6,34)$. The endothelium performs many vital physiological functions, including regulation of blood vessel tone, permeability, hemostasis, as well as synthesis of vascular growth factors $(2,4,6)$. As a result, endothelial function has become critically important in the assessment of cardiovascular diseases (CVD). The ability of blood vessels to vasodilate in 
response to reactive hyperemia secondary to increased shear force requires a healthy endothelium. This response is mediated by the production of nitric oxide (NO) via endothelial NO synthase (eNOS). However, additional mechanisms such as increased concentrations of prostacyclin, endothelium-derived hyperpolarizing factor, and ischemic metabolites may be involved in this response (30). The disruption of NO production and delivery contributes to paradoxical vasoconstriction, which is a hallmark in heart failure (6).

The brachial arteries respond to shear stress induced by increasing blood flow during reactive hyperemia similar to the coronary arteries with a comparable blood vessel caliber. For this reason, they can be used as a surrogate for coronary endothelial function $(7,15)$. Total brachial artery reactivity (TBAR) is a measure of endothelium-dependent vasodilation by ultrasound imaging that indirectly measures NO release in response to shear stress. It relies on obtaining a baseline diameter of the artery following cuff deflation (8). This method compensates for the absence of a stereotactic device limiting the errors associated with ultrasound probe displacement. Polak et al. (8) found TBAR to be an independent predictor of incident coronary heart disease events. However, its association with incident HF and its subtypes in adults free of cardiovascular diseases is currently unknown. Although endothelial dysfunction as measured by brachial flowmediated dilation (FMD) has been associated with incident HF, in particular HFrEF (34), less is known about its role in the development of HFpEF.

The aim of the present study was to investigate whether TBAR is associated with overall incident HF and the two HF sub-types, HF with reduced EF (HFrEF), and HF with preserved EF (HFpEF) in a community-based study of the Multi-Ethnic Study of Atherosclerosis (MESA). We hypothesized that TBAR would be inversely related with incident HF and both HF subtypes. 


\section{METHODS}

\section{Study design and population}

The data for this study came from MESA (9), a continuous, multicenter prospective cohort study of CVD sponsored by the National Heart Lung and Blood Institute of the National Institutes of Health. MESA is a diverse, population-based sample of 6,814 men and women aged 45-84, with no history of clinical CVD at baseline (10). The cohort includes Caucasian, African American, Hispanic, and Asian participants recruited from six field centers across the United States between July 2000 and August 2002. Six additional exams have been completed since 2000, and participants are contacted every 9 to 12 months to assess clinical morbidity and mortality. Specific information on the MESA protocol has been described elsewhere $(9,10,11)$, and additional information can be found at https://www.mesa-nhlbi.org/.

For the present study, the sample included 5,499 participants (45-84 years of age) with available brachial artery endothelial function assessed by ultrasound at the baseline visit (Exam 1). Exclusions included individuals with systolic blood pressures (SBP) $>180 \mathrm{mmHg}$, a blood pressure difference between both arms $\geq 15 \mathrm{mmHg}$ that may be indicative of subclavian stenosis, individuals with previous radical mastectomy, congenital abnormality of the right hand or arm, Raynaud's phenomenon, and technical difficulties. Digital copies of brachial artery diameter responses were not available in 623 cases, leading to 5,499 participants being analyzed (8). The use of MESA data was approved by the Institutional Review Board of the University of North Florida. 


\section{Total brachial artery reactivity}

Participants preparation included abstaining from food and caffeine for six hours prior to the brachial artery endothelial function assessment. If necessary, a small snack, mostly carbohydrates and no fat content was provided 90 minutes prior to the assessment. Consumption of vitamin $\mathrm{C}$ or $\mathrm{E}$ in the six hours before the procedure was discouraged, and the participant should not have smoked any cigarettes in the last six hours (11). Blood pressure was then obtained in both the right and left arms using the automated sphygmomanometer Dinamap Monitor Pro $100 \AA$ (Critikon, Tampa, FL).

Trained technicians at each of the six field centers acquired B-mode ultrasound images with a Logiq-700 ultrasound device (GE Healthcare, Waukesha, WI) and an ultrasound transducer (M12L) set at $9 \mathrm{MHz}$, without the use of a stereotactic holder. Participants were in a supine position during the examination with the right arm supported at the elbow and wrist. The ultrasound probe was placed on the medial aspect of the right arm a few centimeters above the antecubital fossa with a slight angulation to best visualize the brachial artery. An occlusion cuff was placed on the upper right forearm just below the antecubital fossa and inflated to a pressure of $200 \mathrm{mmHg}$ when the baseline SBP was $<150 \mathrm{mmHg}$, or $50 \mathrm{mmHg}$ above maximal systolic pressure when the baseline SBP was $>150 \mathrm{mmHg}$ but $<180 \mathrm{mmHg}$. The cuff was kept inflated for five minutes with the ultrasound probe held centered over the same brachial artery segment. Images were videotaped and recorded using super VHS tapes, starting 15 seconds before cuff deflation, and continuing for 90 seconds after cuff release.

Images were sent to Tufts Medical Center Ultrasound Reading Center for blinded processing. Digital streams of the brachial artery ultrasound images were acquired from the videotapes at a frame-rate of 30 frames-per-second as MJPEG compressed images (compression 
ratio six to one) using a Pinnacle DC-30 Video board (Corel Inc., Mountain View CA) and a Compaq AP-200 workstation (Compaq Computer Corporation, Houston, TX) equipped with a Pentium III processor (Intel Corporation, Santa Clara, CA). A reader reviewed the images and identified the point at which the blood pressure cuff had been released. The reader then identified an appropriate brachial artery segment and placed a rectangular region of interest on a selected image frame. Customized software was used to calculate the location of the near and far wall media-adventitia interfaces in this region of interest and to generate brachial artery diameter versus time curves without manual editing. These were transferred to Access (Microsoft, Redmond WA) databases for archiving. The archived brachial artery diameter curves were subsequently retrieved and processed using a MATLAB (The MathWorks Inc., Natick MA) program that smoothed the diameter versus time curves using a finite impulse response digital filter and processed the resultant curves to identify the maximum diameter and time to maximum diameter starting 20 seconds following release of the blood pressure cuff. The algorithm then searched for a minimum diameter going backwards until 10 seconds after cuff release based on previous observations. Then TBAR was calculated as:

\section{$\operatorname{TBAR}(\%)=\underline{(\text { maximum diameter }- \text { minimum diameter })} \times 100$ (minimum diameter)}

Although 5,499 brachial diameter curves were processed, there were 765 instances where the curve analysis algorithm detected a maximum diameter in a time window 20 to 30 seconds after cuff release and failed to find a smaller diameter in the preceding time interval. These cases likely represented low amplitude responses and were assigned a $0 \%$ TBAR value as previously described (8). 


\section{Heart failure}

Participants were contacted by telephone every six to nine months after the baseline MESA exam to determine whether any medical events have occurred. MESA identifies HF events in both inpatient and outpatient through participant self-report via follow-up calls, notification at clinic visits or directly to the field center. At least two physicians review medical records and death certificates of the eligible events to assign a final classification. HF events can be classified as 'Definite', 'Probable', or 'Absent'. Probable HF requires the presence of HF symptoms, a physician diagnosis of $\mathrm{HF}$, and evidence of the patient receiving medical treatment such as diuretics, vasodilators, beta-blockers, or ACE inhibitors. Definite HF requires the same criteria as probable $\mathrm{HF}$, and one or more of the following criterion: pulmonary edema/congestion, dilated ventricle or poor left ventricular function, or evidence of left ventricular diastolic dysfunction. Those with only HF diagnosis without any treatment or evidence on imaging are classified as absent. Disagreement or conflicting endpoint diagnosis are assigned to a third reviewer, or if necessary, to the MESA morbidity and mortality committee for the final decision. For those participants with a final classification of $\mathrm{HF}$, ejection fraction is recorded either as a percent or classified as 'Normal', 'Low', or 'Unknown'. For this study, incident HF overall included participants with either definite or probable HF, independent of ejection fraction. Participants with $\mathrm{EF} \leq 40 \%$ or classified as 'Low' were considered HFrEF, and those with $\mathrm{EF} \geq 50 \%$ or classified as 'Normal' were considered HFpEF.

\section{Covariates}

Age, sex, race/ethnicity, smoking status, and medical history were self-reported during the baseline MESA exam. The smoking status classified participants as never smokers, former smokers, or current smokers (smoking cigarettes in the last 30 days). Body mass index (BMI) was 
calculated as body weight in kilograms $(\mathrm{kg})$ divided by height in meter squared $\left(\mathrm{m}^{2}\right)$. The four BMI categories used in this study included normal $\left(<25 \mathrm{~kg} / \mathrm{m}^{2}\right)$, overweight $\left(\geq 25-<30 \mathrm{~kg} / \mathrm{m}^{2}\right)$, obese $\left(\geq 30-\leq 40 \mathrm{~kg} / \mathrm{m}^{2}\right)$, and extreme obese $\left(>40 \mathrm{~kg} / \mathrm{m}^{2}\right)$. Blood pressure was measured in the seated position using a Dinamap Monitor Pro $100 \circledR$ (Critikon, Tampa, FL) automated oscillometric device. Blood pressures were the average of the last two of three performed measurements and were categorized in three groups: normal (SBP $<120 \mathrm{mmHg}$ and DBP $<80$ $\mathrm{mmHg}$ ), elevated (SBP 120-129 $\mathrm{mmHg}$ and DBP $<80 \mathrm{mmHg}$ ), and hypertensive (SBP $\geq 130$ $\mathrm{mmHg}$ or DBP $\geq 80 \mathrm{mmHg}$ ). Participants who reported using antihypertensive medications were also considered as being hypertensive. Lipids and lipoproteins categories were determined using the National Cholesterol Education Program 2001 Guidelines (13). Triglyceride values $\geq 150$ $\mathrm{mg} / \mathrm{dL}$ and low-density lipoprotein cholesterol (LDL-C) values $\geq 130 \mathrm{mg} / \mathrm{dL}$ were classified as elevated. High-density lipoprotein cholesterol (HDL-C) values $<50 \mathrm{mg} / \mathrm{dL}$ for females and $<40$ $\mathrm{mg} / \mathrm{dL}$ for males were classified as low. Participants who reported using lipid-lowering medications were also considered as having elevated lipid levels. Waist circumference values $\geq 88$ $\mathrm{cm}$ for females and $\geq 102 \mathrm{~cm}$ for males were considered as being at risk. The presence of diabetes mellitus was based on self-reported physician diagnosis, use of insulin or oral hypoglycemic medication, or a fasting glucose value $\geq 126 \mathrm{mg} / \mathrm{dL}$. Myocardial infarction events that occurred after baseline were self-reported at clinic visits, directly to the field center, or through interim follow-up telephone calls. In addition, events were identified through review and abstraction of medical records and death certificates. Atrial fibrillation diagnosis was self-reported or through review of in-hospital events records via the International Classification of Diseases (ICD9) code (427.31: AF) during follow-up. 


\section{Statistical analyses}

The data in this study were managed using SAS 9.4 (SAS Institute Inc, Cary, NC) (14). Variables recodes, data coding validation, and analysis were conducted in SAS. Variables are presented as means and standard deviation (SD) if continuous and as percentages if categorical. A dichotomous TBAR variable was created based on the median value of the entire sample at baseline (below or above 7.9\%). Analysis was performed after excluding potential TBAR outliers. We set two limits, a minimum value at $0 \%$, and a maximum value at the $99^{\text {th }}$ percentile $(40.9 \%)$. Cox proportional hazards regression models were used to calculate multivariable adjusted hazard ratios (HRs) and 95\% confidence intervals (CI) to determine the risk of HF overall, HFrEF, and HFpEF according to TBAR values. Heart failure participants without EF data were excluded from the subtype analyses. Three different models were created controlling for known HF risk factors including age, sex, race/ethnicity, BMI, diabetes, blood pressure, HDL-C, LDL-C, triglycerides, smoking status, myocardial infarction, and atrial fibrillation. Model 1 was unadjusted, model 2 was age-adjusted, and model 3 fully adjusted. The level of significance was set at $p=0.05$ for all tests. Additionally, using proportional hazards regression, covariates included in the fully adjusted model were chosen using a stepwise backward elimination process; covariates that did not contribute significantly based on $\mathrm{p}=0.05$ were removed and excluded from the final analysis. A final parsimonious model was included to elucidate the relationship between the independent and dependent variables. Kaplan-Meier survival curves were plotted by the median value of TBAR for HF overall, HFrEF, and HFpEF, and compared statistically using the log rank test. Finally, we evaluated the effect of TBAR as a continuous variable (per 1 SD value of 9.7\%) on time to HF, using a cut point of $50 \%$ for EF. The analysis in this study included the whole sample of 5,499 
participants with available TBAR values. We also performed the analysis excluding the 765 curves with low amplitude responses for a full sample of 4,734 participants (data not shown).

\section{RESULTS}

Of the 5,499 participants with available TBAR values, over a mean follow-up period of 12.5 years, incident HF was diagnosed in 250 participants; 98 classified as HFrEF, 106 as HFpEF, and 46 with unknown or borderline EF (41-49\%). Sample characteristics by HF subtypes are shown in Table 1.

\begin{tabular}{|c|c|c|c|c|}
\hline Total $(n=5,499)$ & $\begin{array}{l}\text { Overall HF } \\
\text { n }(\%)\end{array}$ & $\begin{array}{l}\text { HFrEF } \\
\text { n }(\%)\end{array}$ & $\begin{array}{l}\text { HFpEF } \\
\text { n }(\%)\end{array}$ & $\begin{array}{l}\text { No HF } \\
\text { n }(\%)\end{array}$ \\
\hline Total & $250(4.5)$ & $98(48)$ & $106(52)$ & $5,249(95.5)$ \\
\hline Age, Mean (SD) & $68.2(8.93)$ & $67.4(9.19)$ & $68.9(9.08)$ & $61.7(10.18)$ \\
\hline \multicolumn{5}{|l|}{ Sex } \\
\hline Male & $151(60.4)$ & $70(71.4)$ & $57(53.8)$ & $2,548(48.5)$ \\
\hline Female & $99(39.6)$ & $28(28.6)$ & $49(46.2)$ & $2,701(51.5)$ \\
\hline \multicolumn{5}{|l|}{ Race/Ethnicity } \\
\hline Caucasian & $98(39.2)$ & $38(38.8)$ & $42(39.6)$ & $1,898(36.2)$ \\
\hline Asian & $22(8.8)$ & $2(2.0)$ & $13(12.3)$ & $714(13.6)$ \\
\hline African American & $77(30.8)$ & $36(36.7)$ & $29(27.4)$ & $1,452(27.7)$ \\
\hline Hispanic & $53(21.2)$ & $22(22.5)$ & $22(20.8)$ & $1,185(22.6)$ \\
\hline \multicolumn{5}{|l|}{ BMI } \\
\hline Normal & $50(20.0)$ & $22(22.5)$ & $22(20.8)$ & $1,579(30.1)$ \\
\hline Overweight & $108(43.2)$ & $42(42.9)$ & $46(43.4)$ & $2,070(39.4)$ \\
\hline Obese & $80(32.0)$ & $31(31.6)$ & $33(31.1)$ & $1,441(27.5)$ \\
\hline Extreme Obese & $12(4.8)$ & $3(3.1)$ & $5(4.7)$ & $159(3.0)$ \\
\hline
\end{tabular}


Blood pressure

$\begin{array}{lllll}\text { Normal } & 29(11.6) & 15(15.3) & 9(8.5) & 1,804(34.4) \\ \text { Elevated } & 17(6.8) & 5(5.1) & 11(10.4) & 434(8.3) \\ \text { Hypertensive } & 204(81.6) & 78(79.6) & 86(81.1) & 3,011(57.4)\end{array}$

Smoking Status

$\begin{array}{lllll}\text { Never Smoked } & 111(44.4) & 42(42.9) & 43(40.6) & 2,698(51.4) \\ \text { Former Smoker } & 106(42.4) & 39(39.8) & 49(46.2) & 1,897(36.1) \\ \text { Current Smoker } & 33(13.2) & 17(17.4) & 14(13.2) & 654(12.5)\end{array}$

Lipid/Lipoprotein

Elevated LDL-C $110(44.0) \quad 44(44.9) \quad 46(43.4) \quad 2,393(45.6)$

$\begin{array}{lllll}\text { Elevated TG } & 118(47.2) & 42(42.9) & 50(47.2) & 2,047(39.0)\end{array}$

Low HDL-C $\quad 127(50.8) \quad 50(51.0) \quad 46(43.4) \quad 2,409(45.9)$

WC

$\begin{array}{lllll}\text { Healthy } & 93(37.2) & 43(43.9) & 35(33.0) & 2,542(48.4) \\ \text { At risk } & 157(62.8) & 55(56.1) & 71(67.0) & 2,707(51.6)\end{array}$

Diabetes

\begin{tabular}{ccccc} 
No & $177(70.8)$ & $75(76.5)$ & $72(67.9)$ & $4,608(87.8)$ \\
Yes & $73(29.2)$ & $23(23.5)$ & $34(32.1)$ & $640(12.2)$ \\
TBAR & & & \\
$\geq 7.9$ & $100(40.0)$ & $37(37.8)$ & $43(40.6)$ & $2,595(49.4)$ \\
$<7.9$ & $150(60.0)$ & $61(62.2)$ & $63(59.4)$ & $2,654(50.6)$ \\
MI & $221(88.4)$ & $88(89.8)$ & $94(88.7)$ & $5,116(97.5)$ \\
No & $29(11.6)$ & $10(10.2)$ & $12(11.3)$ & $133(2.5)$ \\
Yes & & & \\
AF & $210(84.0)$ & $87(88.8)$ & $83(78.3)$ & $5,026(95.8)$ \\
No & $40(16.0)$ & $11(11.2)$ & $23(21.7)$ & $223(4.2)$ \\
Yes & & & & \\
\hline
\end{tabular}


$\mathrm{HF}$, heart failure; HFrEF, heart failure with reduced ejection fraction ( $\mathrm{EF} \leq 40 \%$ or 'Low'); $\mathrm{HFpEF}$, heart failure with preserved ejection fraction ( $\mathrm{EF} \geq 50 \%$ or 'Normal'); BMI, body mass index; LDL-C, low-density lipoprotein cholesterol; HDL-C, high-density lipoprotein cholesterol; TG, triglycerides; WC, waist circumference; TBAR, total brachial artery reactivity; MI, myocardial infarction; AF, atrial fibrillation.

*HF participants with unknown or borderline EF (41-49\%) were excluded from the subtype analyses $(n=46)$.

The participants with HF were significantly older than those without $\mathrm{HF}(\mathrm{p}<0.001)$. The prevalence of HF overall and HFrEF were higher in males $(p<0.001)$ than in females, but the same was not seen in those with HFpEF. Additionally, there was a greater proportion of diabetes, hypertension, myocardial infarction, and atrial fibrillation in those with HF, regardless of subtype $(\mathrm{p}<0.01)$. The proportion of participants with waist circumference values considered at risk was significantly greater in those with HF and HFpEF $(\mathrm{p}<0.01)$. There was a slightly but significantly greater proportion of TBAR values below the median in those with $\mathrm{HF}$ and $\operatorname{HFrEF}(\mathrm{p}<0.01$ and $\mathrm{p}<0.05$, respectively). However, there were no significant differences between the mean values of TBAR for HFrEF and HFpEF (8.09 \pm 6.60 vs $8.56 \pm 7.79, \mathrm{p}=0.64$, respectively).

\section{Risk of Heart Failure}

The results of the multivariable adjusted model using the proportional hazard regression procedure for HF overall are shown in Table 2. Crude analysis revealed that those with TBAR below the median (7.9\%) had significantly higher risk of HF (HR 1.46; 95\% CI 1.13-1.88, p $<0.01$ ). In the age-adjusted model, the risk of HF was attenuated but remained statistically significant (HR $1.30 ; 95 \%$ CI 1.01-1.68, $\mathrm{p}<0.05)$. However, in the fully adjusted model, these relationships were no longer significant $(\mathrm{p}=0.79)$. 
Table 2. Hazard ratios (HR) associated with total brachial artery reactivity $<7.9 \%$ and risk of incident heart failure

Heart Failure Overall $(n=250)$

\begin{tabular}{|c|c|c|}
\hline Model & HR & $95 \% \mathrm{CI}$ \\
\hline 1 & $1.46 * *$ & $1.13-1.88$ \\
\hline 2 & $1.30^{*}$ & $1.01-1.68$ \\
\hline 3 & 1.23 & $0.95-1.59$ \\
\hline \multicolumn{3}{|c|}{$\begin{array}{l}\text { Model 1: unadjusted; model 2: adjusted by age; model 3: adjusted by sex, race/ethnicity, BMI, } \\
\text { diabetes, blood pressure, HDL-C, LDL-C, triglycerides, smoking status, myocardial infarction, } \\
\text { and atrial fibrillation. CI, confidence interval. }{ }^{*} \mathrm{p}<0.01 ; * \mathrm{p}<0.05 \text {. }\end{array}$} \\
\hline \multicolumn{3}{|c|}{ Table 3 shows the HRs of HF overall in the parsimonious model. In this sample, from all } \\
\hline \multicolumn{3}{|c|}{ the covariates, sex, diabetes, blood pressure, myocardial infarction, and atrial fibrillation were } \\
\hline \multicolumn{3}{|c|}{ significantly associated with incident HF. Males had a significantly higher risk of HF overall } \\
\hline \multicolumn{3}{|c|}{ compared with females (HR 1.42; 95\% CI 1.10-1.84, $\mathrm{p}<0.01$ ). Having diabetes, elevated blood } \\
\hline \multicolumn{3}{|c|}{ pressure or being hypertensive resulted in more than twice elevated risk of HF. The presence of a } \\
\hline \multicolumn{3}{|c|}{ previous event of myocardial infarction or atrial fibrillation resulted in a two to three-time greater } \\
\hline \multicolumn{3}{|c|}{ risk of HF overall (HR 2.98; 95\% CI 2.01-4.44 and HR 3.07; 95\% CI 2.18-4.34, p<0.001, } \\
\hline
\end{tabular}


Table 3. Hazard ratios (HR) associated with risk of incident heart failure in the parsimonious model

Heart Failure Overall $(n=250)$

\begin{tabular}{lll}
\hline & HR & 95\% CI \\
Sex (male) & $1.42^{*}$ & $1.10-1.84$ \\
Diabetes (yes) & $2.48^{* *}$ & $1.88-3.28$ \\
Blood pressure & & \\
$\quad$ Normal & & \\
Elevated & $2.34^{*}$ & $1.28-4.26$ \\
Hypertensive & $3.49^{* *}$ & $2.36-5.18$ \\
MI (yes) & $2.98^{* *}$ & $2.01-4.44$ \\
AF (yes) & $3.07^{* *}$ & $2.18-4.34$ \\
\hline MI, myocardial infarction; AF, atrial fibrillation; CI, confidence interval. $* * \mathrm{p}<0.001 ; * \mathrm{p}<0.01$.
\end{tabular}

Figure 1 displays the Kaplan-Meier Survival curve showing the likelihood of remaining HF free with time according to TBAR values. Results indicate significantly lower risk of developing HF in those with TBAR above the median (log-rank $\mathrm{p}<0.05)$. When examining TBAR as a continuous variable, every 1 -standard deviation $(9.7 \%)$ increase in TBAR resulted in a $19 \%$ decrease in risk of HF overall $(\mathrm{p}<0.05)$. This corresponds to a $2 \%$ decrease in risk for each percent increase in TBAR. Similar results were obtained when we repeated the analysis excluding the 765 curves with low amplitude responses (data not shown). 
Figure 1. Kaplan-Meier Survival curves showing the likelihood of remaining heart failure free with time according to total brachial artery reactivity values

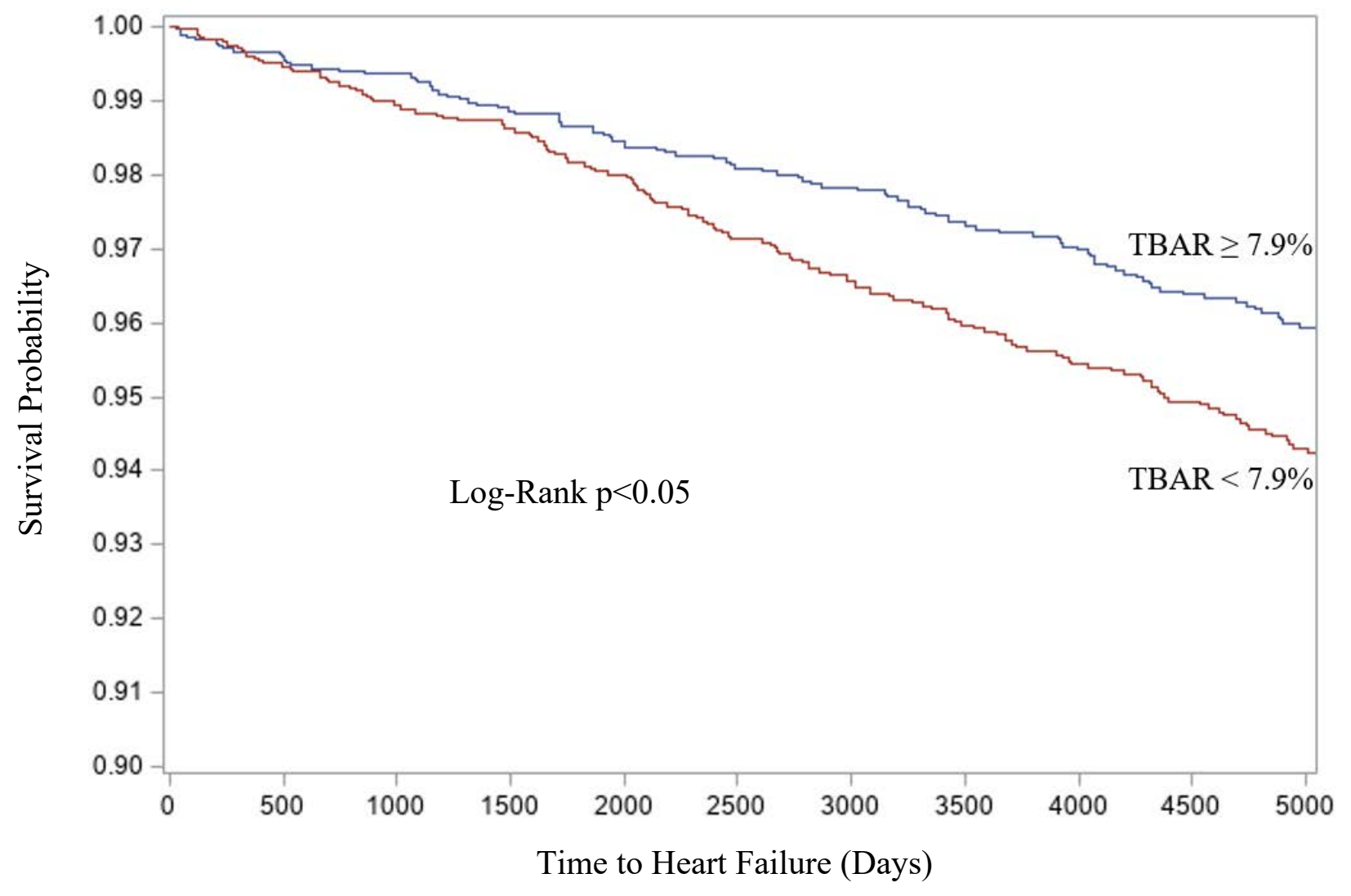

*TBAR, total brachial artery reactivity.

\section{Risk of Heart Failure with Reduced Ejection Fraction}

Table 4 displays the results of the proportional hazard regression analyses for HFrEF. Crude analysis revealed that those with TBAR below the median had a significantly greater risk of HFrEF (HR 1.61; 95\% CI 1.07-2.43, p<0.05). In the age-adjusted model, the risk of HFrEF was attenuated and was borderline significant $(\mathrm{p}=0.06)$. In the fully adjusted model, these relationships did not reach significance. 
Table 4. Hazard ratios (HR) associated with total brachial artery reactivity $<7.9 \%$ and risk of incident heart failure with reduced ejection fraction

\begin{tabular}{|c|c|c|}
\hline \multicolumn{3}{|c|}{ HFrEF (n=98) } \\
\hline Model & HR & $95 \% \mathrm{CI}$ \\
\hline 1 & $1.61 *$ & $1.07-2.43$ \\
\hline 2 & 1.46 & $0.97-2.20$ \\
\hline 3 & 1.36 & $0.90-2.07$ \\
\hline \multicolumn{3}{|c|}{$\begin{array}{l}\text { Model 1: unadjusted; model 2: adjusted by age; model 3: adjusted by sex, race/ethnicity, BMI, } \\
\text { diabetes, blood pressure, HDL-C, LDL-C, triglycerides, smoking status, myocardial infarction, } \\
\text { and atrial fibrillation. CI, confidence interval. }{ }^{*} \mathrm{p}<0.05 \text {. }\end{array}$} \\
\hline \multicolumn{3}{|c|}{ Table 5 shows the HRs of HFrEF in the parsimonious model. Diabetes, sex, race/ethnicity, } \\
\hline \multicolumn{3}{|c|}{ blood pressure, myocardial infarction, and atrial fibrillation were significantly associated with } \\
\hline \multicolumn{3}{|c|}{ incident HFrEF. Males had significantly greater risk of HFrEF compared with females (HR 2.40; } \\
\hline \multicolumn{3}{|c|}{$95 \%$ CI $1.54-3.75, \mathrm{p}<0.001)$. Although there was a $10 \%$ greater risk of HFrEF in African } \\
\hline \multicolumn{3}{|c|}{ Americans, the results were not statistically significant. Having diabetes was associated with a } \\
\hline \multicolumn{3}{|c|}{ greater risk of HFrEF and being hypertensive at baseline resulted in more than a two-fold increase } \\
\hline \multicolumn{3}{|c|}{ in risk of HFrEF $(p<0.01)$. The presence of a previous event of myocardial infarction or atrial } \\
\hline
\end{tabular}


Table 5. Hazard ratios (HR) associated with risk of incident heart failure with reduced ejection fraction in the parsimonious model

\section{HFrEF (n=98)}

\begin{tabular}{|c|c|c|}
\hline & HR & $95 \% \mathrm{CI}$ \\
\hline Sex (male) & $2.40 * * *$ & $1.54-3.75$ \\
\hline Diabetes (yes) & $1.87 * *$ & $1.14-3.05$ \\
\hline \multicolumn{3}{|l|}{ Race/ethnicity } \\
\hline \multicolumn{3}{|l|}{ Caucasian } \\
\hline African A. & 1.10 & $0.69-1.77$ \\
\hline Asian & $0.14 * *$ & $0.03-0.60$ \\
\hline Hispanic & 0.84 & $0.49-1.46$ \\
\hline \multicolumn{3}{|l|}{ Blood pressure } \\
\hline \multicolumn{3}{|l|}{ Normal } \\
\hline Elevated & 1.32 & $0.48-3.63$ \\
\hline Hypertensive & $2.58 * *$ & $1.46-4.53$ \\
\hline MI (yes) & $2.71 * *$ & $1.38-5.31$ \\
\hline AF (yes) & $2.06^{*}$ & $1.08-3.91$ \\
\hline
\end{tabular}

Figure 2 displays the Kaplan-Meier Survival curve showing the likelihood of remaining HFrEF free with time according to TBAR values. Results indicate significantly lower risk of developing HFrEF in those with TBAR above the median (log-rank $\mathrm{p}=0.05$ ). When examining TBAR as a continuous variable, with a cut point of 50\% for EF, every 1 -standard deviation (9.7\%) increase in TBAR resulted in a $29 \%$ decrease in risk of HFrEF $(p=0.05)$. This corresponds to a $3 \%$ decrease in risk for each percent increase in TBAR. 
Figure 2. Kaplan-Meier Survival curves showing the likelihood of remaining heart failure with reduced ejection fraction free with time according to total brachial artery reactivity values

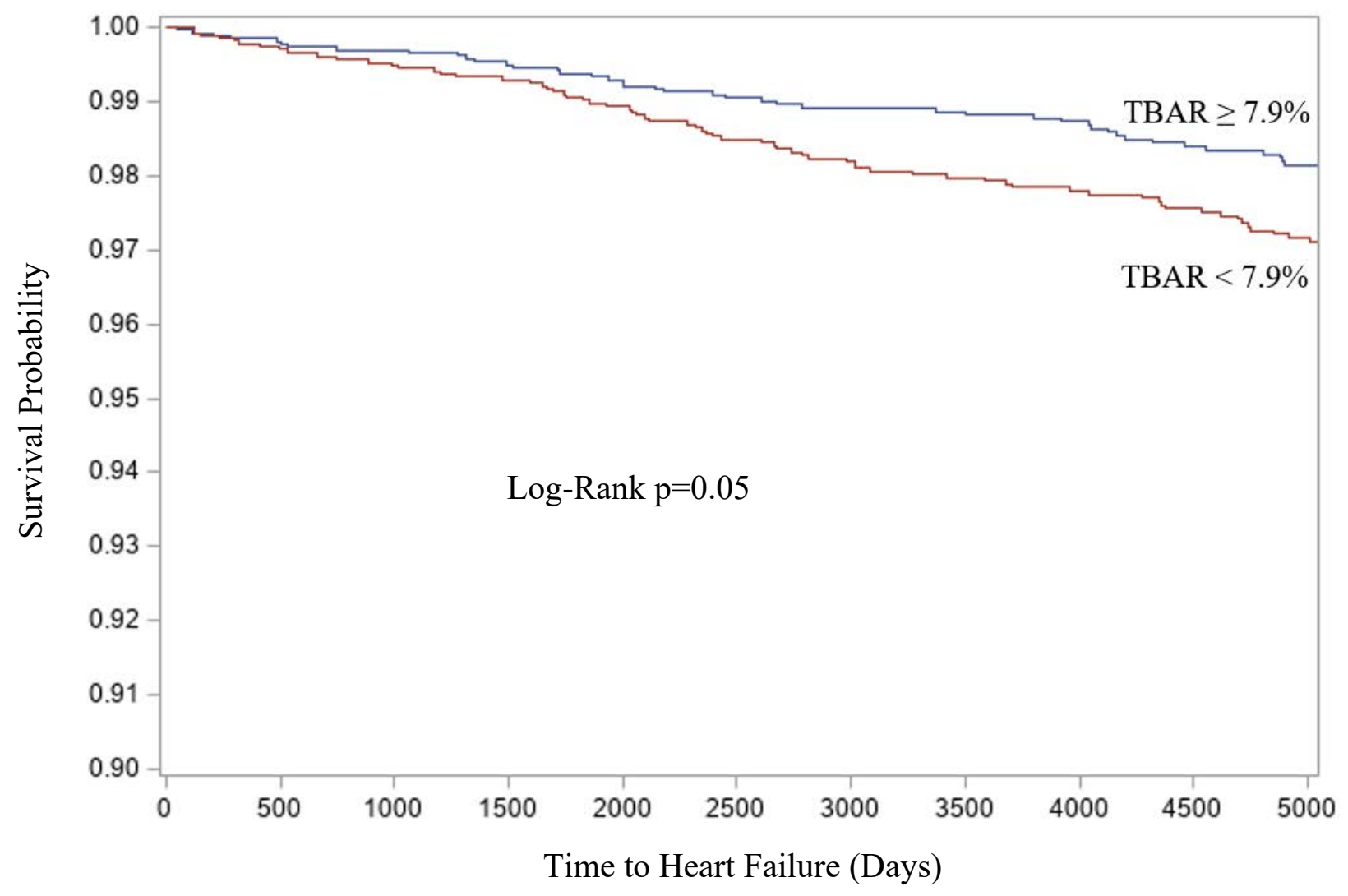

*TBAR, total brachial artery reactivity.

\section{Risk of Heart Failure with Preserved Ejection Fraction}

Table 6 displays the results of the proportional hazard regression procedure for HFpEF.

Crude analysis revealed borderline significant results in those with HFpEF (HR 1.43; 95\% CI 0.97-

2.12, $\mathrm{p}=0.06$ ). 
Table 6. Hazard ratios (HR) associated with total brachial artery reactivity $<7.9 \%$ and risk of incident heart failure with preserved ejection fraction

\begin{tabular}{lcc}
\hline & HFpEF (n=106) \\
\hline Model & $1.43^{*}$ & 95\% CI \\
1 & 1.28 & $0.97-2.12$ \\
2 & 1.19 & $0.86-1.88$ \\
3 & $0.80-1.77$ \\
\hline $\begin{array}{l}\text { Model 1: unadjusted; model 2: adjusted by age; model 3: adjusted by sex, race/ethnicity, BMI, } \\
\text { diabetes, blood pressure, HDL-C, LDL-C, triglycerides, smoking status, myocardial infarction, }\end{array}$ \\
and atrial fibrillation. CI, confidence interval. *p=0.06. \\
$\begin{array}{l}\text { Table } 7 \text { shows the HRs of HFpEF in the parsimonious model. Diabetes, blood pressure, } \\
\text { myocardial infarction, and atrial fibrillation were significantly associated with incident HFpEF. }\end{array}$ \\
Having diabetes was associated with more than two-fold increase in the risk of HFpEF (HR 2.92; \\
95\% CI 1.93-4.42, p<0.001). Those who had elevated blood pressure or were hypertensive at \\
myocardial infarction or atrial fibrillation resulted in a three to four-fold greater risk of HFpEF.
\end{tabular}


Table 7. Hazard ratios (HR) associated with risk of incident heart failure with preserved ejection fraction in the parsimonious model

\section{HFpEF (n=106)}

\begin{tabular}{lll}
\hline & HR & 95\% CI \\
Diabetes (yes) & $2.92^{*}$ & $1.93-4.42$ \\
Blood pressure & & \\
$\quad$ Normal & & \\
Elevated & $4.80^{*}$ & $1.98-11.59$ \\
Hypertensive & $4.60^{*}$ & $2.30-9.20$ \\
MI (yes) & $3.00^{*}$ & $1.63-5.51$ \\
AF (yes) & $4.71 *$ & $2.94-7.54$ \\
\hline MI, myocardial infarction; AF, atrial fibrillation; CI, confidence interval. ${ }^{*} \mathrm{p}<0.001$.
\end{tabular}

Figure 3 displays the Kaplan-Meier Survival curve showing the likelihood of remaining HFpEF free with time according to TBAR values. Results suggest a borderline significant lower risk of developing HFpEF in those with TBAR above the median (log-rank $p=0.06$ ). When examining TBAR as a continuous variable, results did not reach statistically significance. 
Figure 3. Kaplan-Meier Survival curves showing the likelihood of remaining heart failure with preserved ejection fraction free with time according to total brachial artery reactivity values

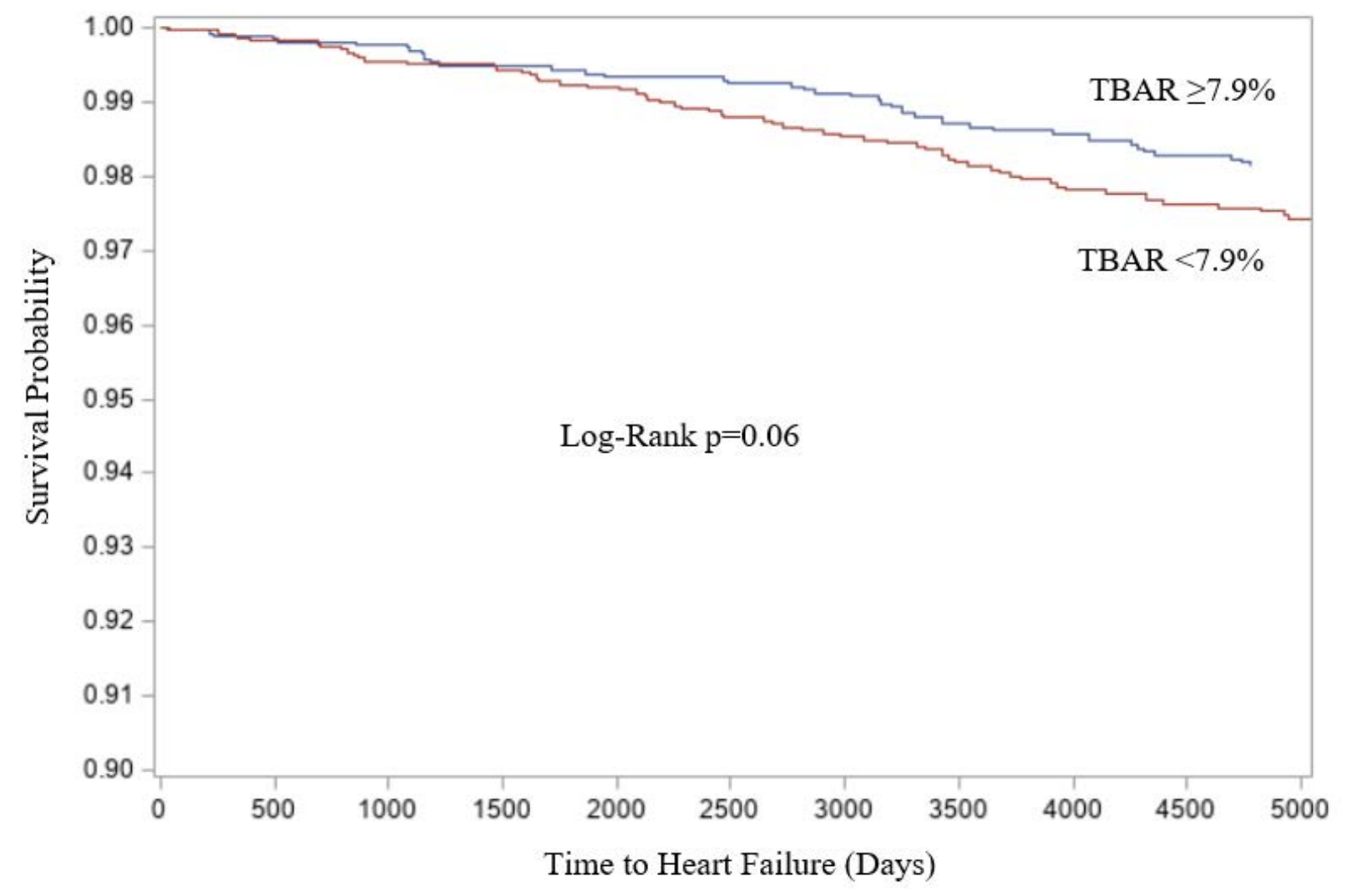

\section{DISCUSSION}

In this population-based study, lower TBAR values were associated with higher rates of incident HF and HFrEF, and borderline significant findings were revealed in those with HFpEF. Furthermore, when examined as a continuous variable, every 1-SD (9.7\%) increase in TBAR resulted in a $19 \%$ and $29 \%$ decrease in risk of $\mathrm{HF}(\mathrm{p}<0.05)$ and $\operatorname{HFrEF}(\mathrm{p}=0.05)$, respectively. These findings suggest a possible role of endothelial dysfunction in HF pathogenesis and add to the evidence demonstrating that assessment of endothelial function is essential in diagnosis and treatment of cardiovascular disease $(2,4,6,31)$. 
Noninvasive assessment of endothelial function is commonly conducted using the traditional FMD technique. Several studies have found an association between endothelial dysfunction measured by FMD and incident cardiovascular disease (CVD) events (7, 16, 17, 18). The MESA study conducted by Yeboah et al. (18), demonstrated that endothelial dysfunction measured by FMD of the brachial artery is associated with a higher rate of incident adverse CVD events during a five-year follow-up period. The meta-analysis of Inaba et al. (6) involving 5,547 participants, found that impairment of brachial FMD is significantly associated with future CVD events. The pooled relative risks of CVD events per $1 \%$ increase in brachial FMD following adjustment for confounding risk factors was 0.87 , corresponding to a $13 \%$ lower risk.

A uniqueness of the present study was the use of TBAR, that relies on baseline brachial artery diameters obtained after the release of a blood pressure occlusion cuff, and its association with incident $\mathrm{HF}$ and its subtypes. Although the measurement process is different from the traditional FMD, they might be comparable and equivalent. Ostrem et al. (19) compared preocclusion, occlusion, and post-occlusion baseline brachial artery measurements on the calculation of FMD in 418 children and 533 adults. They found no significant differences between the three baseline measurements of brachial artery diameter. Additionally, peak FMD values were not significantly different when calculated from pre-occlusion, occlusion or post-occlusion baseline diameters in both children and adults. In agreement, Polak et al. (8) compared TBAR and FMD data from MESA and found statistical equivalence between them.

Our findings are in agreement with the previous study of Ärnlöv et al. (34) using FMD as a predictor of incident HF. This study revealed that individuals in the highest quartile of FMD had a $47 \%$ lower HF risk compared with individuals in the lowest quartile. When analyzing by HF subtypes, they reported lower risk for HFrEF in those individuals with higher FMD values, but the 
same was not seen in those with HFpEF (HR 0.79, 95\% CI 0.64-0.97 vs. $0.99,95 \%$ CI $0.78-1.26$, respectively). Similarly, Polak et al. (8) reported that increased TBAR values were associated with lower risk of incident coronary heart disease (CHD) events with a HR of 0.78 per SD increase. In addition, they found that a TBAR above the median of $7.87 \%$ was associated with a $31 \%$ lower risk of CHD events (HR 0.69; 95\% CI 0.55-0.87, $\mathrm{p}=0.001$ ).

Although we found significantly greater risk of HF and HFrEF, and borderline significant higher risk of HFpEF in those with TBAR below the median, these relationships were no longer statistically significant following adjustment for known HF risk factors. These results suggest that some coronary risk factors are independently related to impaired endothelial function and they may mediate this relationship. Several studies have examined the association between coronary risk factors and impaired endothelial function. Hashimoto et al. (21) found that endothelial function in subjects without coronary risk factors (i.e., hyperlipidemia, diabetes, hypertension, and cigarette smoking) was significantly greater than that in subjects with one or more risk factors. Endothelial dysfunction appears to result from reduced levels of NO bioavailability largely related to baseline coronary risk factors (31). This association is attributed to high oxidative stress characterized by an increase in reactive oxygen species that scavenge and degrade available NO within the vascular wall, and inflammation $(22,15)$.

Parsimonious models in this study indicated that diabetes, hypertension, myocardial infarction, and atrial fibrillation were significantly associated with incident HF, HFrEF, and HFpEF. Male sex was associated with higher risk of HF and HFrEF, but not with HFpEF. Lee et al. (12) evaluated the differential impact of various demographic characteristics and comorbid conditions on the development of HFrEF and HFpEF among Medicare beneficiaries. The most influential predictors for HFrEF were male sex, diabetes, and myocardial infarction; and for 
HFpEF atrial fibrillation and diabetes were the most influential comorbidities (relative influence of $8.4 \%$ and $5.6 \%$, respectively). Similarly, the risk of HF was three-times greater in those with CHD and two-times greater in those with diabetes (20). The population attributable risk was highest for CHD with a greater proportion in men, and hypertension with a greater proportion in women. Celermajer et al. (23) reported that in 238 subjects with no known cardiovascular risk factors aging is associated with progressive endothelial dysfunction. The decline appears to occur earlier in men than in women, with a steep decline in women around the time of the menopause ( $0.21 \% /$ year after $40 \mathrm{~s}$, and $0.49 \% /$ year after the early $50 \mathrm{~s}$, respectively). Paniagua et al. (24) found that hypertensive patients had significant impairment of FMD when compared with healthy controls. In contrast, the same was not true for hypercholesterolemic patients. Diabetes has largely been associated with endothelial dysfunction; it seems that the mechanisms behind this association are related with inflammation, insulin-resistance, and hyperglycemia $(25,26)$. In a study of 2,936 participants from MESA, O'neal et al. (27) reported that lower FMD values were associated with increased rates of atrial fibrillation, suggesting a potential role of endothelial dysfunction in its pathogenesis.

The current study is not without limitations. The sample size was relatively small for those with HF, HFrEF and HFpEF, limiting the generalizability of the results. This may have contributed to the borderline significant results observed in HFpEF. It is possible that with a greater sample size, the association between TBAR and HFpEF would have reached statistically significance. In this context, it should be emphasized that TBAR and EF were measured at a single time point. Changes in FMD may be more valuable in risk stratifying than relying in a single FMD measurement at a given point in time (28). In the present study, TBAR was obtained at one visit. In addition, incident HF was defined as time to first HF event, resulting in the exclusion of 
subsequent HF events with potential changes in EF over time. TBAR was measured with an occlusion cuff placed proximal to the ultrasound probe (upper right forearm), which may indicate that the vasodilatory response is not mediated exclusively by NO. Increased blood flow and shear stress secondary to reactive hyperemia can stimulate vasorelaxation by different mechanisms including increased concentrations of prostacyclin, endothelium-derived hyperpolarizing factor, and ischemic metabolites (30). Doshi et al. (32) found that dilation was significantly greater after upper arm occlusion when compared with wrist occlusion. In addition, during intra-arterial infusion of NO synthase inhibitor, dilatation after upper arm occlusion was partially attenuated (from $11.62 \pm 3.17 \%$ to $7.51 \pm 2.34 \%$; $<0.006$ ), whereas dilation after wrist occlusion was abolished (from $7.25 \pm 2.49 \%$ to $0.16 \pm 2.24 \% ; \mathrm{p}<0.001$ ). They concluded that dilatation after upper arm occlusion has a substantial component not mediated by NO, most probably related to tissue ischemia around the brachial artery. However, a study conducted by Vogel et al. (33) found that subjects with and without risk factors (hypercholesterolemia, hypertension, cigarette smoking) are better differentiated regarding endothelial function using the upper arm occlusion when compared to lower arm occlusion. A possible explanation for this, is that vasodilation is greater using the upper arm occlusion as a result of an increased hyperemic flow-mediated stimulation.

In conclusion, the present study demonstrated that lower TBAR values are associated with higher rates of incident $\mathrm{HF}$ and $\mathrm{HFrEF}$, and borderline significance with $\mathrm{HFpEF}$, indicating a possible role of endothelial dysfunction in HF pathogenesis. The impact of other known HF risk factors may mediate this relationship, thus further research with a greater and more heterogenous sample of adults free of CVD is warranted. It is critical to continue validating findings regarding differences in risk factors leading to HFpEF and HFrEF. 


\section{REFERENCES}

1. Heitzer T, Baldus S, Von Kodolitsch Y, et al. Systemic endothelial dysfunction as an early predictor of adverse outcome in heart failure. Arterioscler Thromb Vasc Biol. 2005; 25(6):1174-1179.

2. Alem MM. Endothelial dysfunction in chronic heart failure: assessment, findings, significance, and potential therapeutic targets. Int. J. Mol. Sci. 2019; 20(13):3198.

3. Roger VL. Epidemiology of heart failure. Circ Res. 2013; 113(6): 646-659.

4. Giannitsi S, Mpougiaklh M, Bechlioulis A, Naka K. Endothelial dysfunction and heart failure: A review of the existing bibliography with emphasis on flow mediated dilation. JRSM Cardiovasc Dis. 2019; 8:1-7.

5. Virani SS, Alonso A, Benjamin EJ, et al. Heart disease and stroke statistics-2020 update: a report from the American Heart Association. Circulation. 2020; 9(141): e139-e596.

6. Chong AY, Blann AD, Lip GYH. Assessment of endothelial damage and dysfunction: observations in relation to heart failure. Q J Med. 2003; 96:253-267.

7. Inaba Y, Chen JA, Bergmann SR. Prediction of future cardiovascular outcomes by flowmediated vasodilatation of brachial artery: A meta-analysis. Int J Cardiovasc Imaging. 2010; 26(6):631-640.

8. Polak J, Ouyang P, Vaidya D. Total brachial artery reactivity and first time incident coronary heart disease events in a longitudinal cohort study: The multi-ethnic study of atherosclerosis. PLoS ONE. 2019; 14(4): e0211726.

9. Bild DE, Bluemke DA, Burke GL, et al. Multi-Ethnic Study of Atherosclerosis: objectives and design. Am J Epidemiol. 2002; 156(9):871-881. 
10. MESA overview and protocol. [cited 2021 January]. Available from http://www.mesanhlbi.org/aboutMesaOverviewProtocol.aspx.

11. MESA Coordinating Center. MESA manual of operations: field center and laboratory

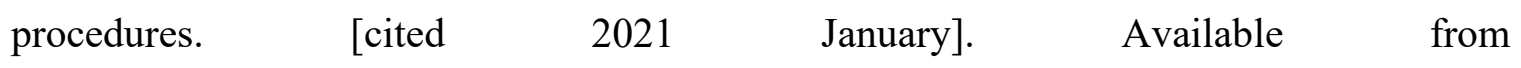
http://www.mesanhlbi.org/publicDocs/MesaMop/MesaMop1-5-01.doc.

12. Lee MP, Glynn RJ, Schneeweiss S, et al. Risk factors for heart failure with preserved or reduced ejection fraction among Medicare beneficiaries: Applications of competing risks analysis and gradient boosted model. Clin Epidemiol. 2019; 12:607-616.

13. National Cholesterol Education Program (NCEP) Expert Panel on detection, evaluation, and treatment of high blood cholesterol in adults (adult treatment panel III). JAMA. 2001; 285(19):2486-97.

14. SAS Institute Inc. 2016. Base SAS ${ }^{\circledR} 9.4$ Procedures Guide: Statistical Procedures, Sixth Edition. Cary, NC: SAS Institute Inc.

15. Patvardhan EA, Heffernan KS, Ruan JM, et al. Assessment of vascular endothelial function with peripheral arterial tonometry: information at your fingertips? Cardiol Rev. 2010; 18(1): 20-28.

16. Ras RT, Streppel MT, Draijer R, et al. Flow-mediated dilation and cardiovascular risk prediction: A systematic review with meta-analysis. Int J Cardiol. 2012; 168:344-351.

17. Shechter M, Issachar A, Marai I, et al. Long-term association of brachial artery flowmediated vasodilation and cardiovascular events in middle-aged subjects with no apparent heart disease. Int J Cardiol. 2009; 134(1):52-58. 
18. Yeboah J, Folsom AR, Burke GL, et al. Predictive value of brachial flow-mediated dilation for incident cardiovascular events in a population-based study: The multi-ethnic study of atherosclerosis. Circulation. 2009; 120(6):502-509.

19. Ostrem JD, Dengel DR, Marlatt KL, et al. Comparison of baseline brachial artery measurements and effect on peak flow-mediated dilation. Clin Physiol Funct Imaging. 2015; 35(1):34-40.

20. Dunlay SM, Weston SA, Jacobsen SJ, et al. Risk factors for heart failure: a populationbased case-control study. Am J Med. 2009; 122(11):1023-1028.

21. Hashimoto M, Kozaki K, Eto M, et al. Association of coronary risk factors and endothelium-dependent flow-mediated dilatation of the brachial artery. Hypertens Res. $2000 ; 23(3): 233-238$.

22. Bauersachs J, Widder JD. Endothelial dysfunction in heart failure. Pharmacol Rep. 2008. 60(1):119-126.

23. Celermajer DS, Sorensen KE, Spiegelhalter DJ, et al. Aging is associated with endothelial dysfunction in healthy men years before the age-related decline in women. J Am Coll Cardiol. 1994; 24:471-476.

24. Paniagua OA, Bryant MB, Panza JA. Role of endothelial nitric oxide in shear stressinduced vasodilation of human microvasculature: diminished activity in hypertensive and hypercholesterolemic patients. Circulation. 2001; 103:1752-1758.

25. Hadi AR, Suwaidi JA. Endothelial dysfunction in diabetes mellitus. Vasc Health Risk Manag. 2007:3(6) 853-876. 
26. Avogaro A, Albiero M, Menegazzo L, De Kreutzenberg S, Fadini GP. Endothelial dysfunction in diabetes. Diabetes care. 2011; 34(2): S285-290.

27. O’Neal WT, Efird JT, Yeboah J, Nazarian S, et al. Brachial flow-mediated dilation and incident atrial fibrillation: the multi-ethnic study of atherosclerosis. Arterioscler Thromb Vasc Biol. 2014; 34(12): 2717-2720.

28. Green DJ, Jones H, Thijssen D, et al. Flow-mediated dilation and cardiovascular event prediction. Does nitric oxide matter? Hypertension. 2011; 57(3):363-369.

29. Loehr LR, Rosamond WD, Chang PP, et al. Heart failure incidence and survival (from the Atherosclerosis Risk in Communities study). Am J Cardiol. 2008; 101:1016-1022.

30. Eskurza I, Seals DR, DeSouza CA, Tanaka H. Pharmacological vs. flow-mediated assessments of peripheral vascular endothelial vasodilatory function in humans. Am J Cardiol. 2001; 88(9): 1067-1069.

31. Widmer RJ, Lerman A. Endothelial dysfunction and cardiovascular disease. Glob Cardiol Sci Pract. 2014; 2014(3):291-308.

32. Doshi SN, Naka KK, Payne N, et al. Flow-mediated dilatation following wrist and upper arm occlusion in humans: the contribution of nitric oxide. Clin Sci. 2001; 101(6):629-635.

33. Vogel RA, Corretti MC, Plotnick GD. A comparison of brachial artery flow-mediated vasodilation using upper and lower arm arterial occlusion in subjects with and without coronary risk factors. Clin Cardiol. 2000; 23(8):571-575. 
34. Ärnlöv J, Sang Y, Ballew SH, et al. Endothelial dysfunction and the risk of heart failure in a community-based study: the Multi-Ethnic Study of Atherosclerosis. ESC Heart Fail. 2020; 7(6):4231-4240. 
Appendices 


\section{Appendix A}

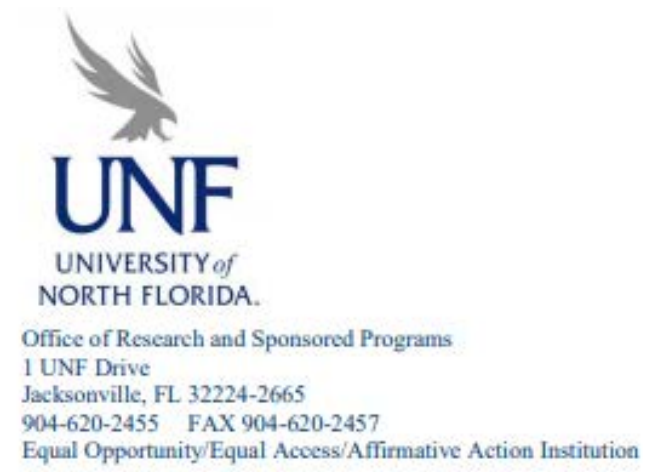

\section{MEMORANDUM}

DATE: February 4, 2020

TO: $\quad$ Ms. Daniela Charry

VIA: Dr. James Churilla

DEPT: $\quad$ Clinical and Applied Movement Science

FROM: Dr. Jennifer Wesely, Chairperson UNF Institutional Review Board

RE: Review conducted on behalf of the UNF Institutional Review Board "Predictive value of Brachial Flow-Mediated Dilation for incident Heart Failure with reduced versus preserved ejection fraction in a population-based study."

This is to advise you that your project, "Predictive value of Brachial Flow-Mediated Dilation for incident Heart Failure with reduced versus preserved ejection fraction in a population-based study," was reviewed on behalf of the UNF Institutional Review Board and was declared "not research involving human subjects" based on the definitions provided in the U.S. Department of Health and Human Services Code of Federal Regulations found at 45 CFR 46.102. As such, this project qualifies for a Waiver of IRB Review.

Please note, this waiver does not absolve the Principal Investigator from complying with other federal, state, or local laws or institutional policies and procedures that may be applicable in the conduct of this project.

This waiver applies to your project in the form and content as submitted to the IRB for review. Any variations or modifications to this project involving the participation of human subjects must be approved by the IRB prior to implementing such changes. Please maintain a copy of this waiver for your records.

Thank you for submitting your project to the IRB for consideration. Should you have any questions or if we can be of further assistance, please contact the Research Integrity office at 904-620-2455, or IRB@unf.edu. 


\section{Vita}

Daniela Charry is an international graduate teaching and research assistant in the Clinical and Applied Movement Sciences department at the University of North Florida in Jacksonville, FL. There, she recently completed her master's degree in Exercise Science and Chronic Disease. Upon earning her Bachelor of Science in Physical Therapy degree from the National University of Colombia in 2013 and graduating with the best GPA of her cohort, she completed an internship at the Intensive Care Unit (ICU) of the Hospital Universitario de la Samaritana. There she conducted a study titled "Early mobilization, duration of mechanical ventilation and stay in intensive care". This research was awarded as the best Physical Therapy degree work in the XXIII version competition of undergrad programs at the National University of Colombia. She worked as a Physical Therapist in the ICU and later in the neonatal intensive care unit for about three years.

Originally from Bogota, Colombia, Daniela currently lives in Jacksonville, FL, where she also plays rugby at the Jacksonville Women's Rugby team. Her research interests consist of heart failure, endothelial function, and acute and chronic exercise responses and adaptations related to cardiovascular health in individuals who are sedentary, aged, or diseased. Her goal is to pursue a Ph.D. in Exercise Physiology at the University of Texas at Austin with the intent of a career in academia and research. 